\title{
Sedimentología e icnología de un delta fluvio-dominado, Formación Lajas (Jurásico Medio), cuenca Neuquina, Argentina
}

\author{
*Nerina Canale', Juan José Ponce ${ }^{1,2}$, Noelia B. Carmona ${ }^{1,2}$, Martín N. Parada1 ${ }^{1}$ Daniel I. Drittanti
}

\author{
${ }^{1}$ Universidad Nacional de Rio Negro. Instituto de Investigación en Paleobiología y Geología. Isidro Lobo 516. Río Negro, Argentina. \\ ncanale@unrn.edu.ar; mnparada@unrn.edu.ar \\ ${ }^{2}$ Consejo Nacional de Investigaciones Cientificas y Técnicas. Instituto de Investigación en Paleobiología y Geología. Av. Julio A. \\ Roca 1242, Río Negro, Argentina. \\ jponce@unrn.edu.ar; ncarmona@unrn.edu.ar \\ ${ }^{3}$ Departamento de Geología, Universidad Nacional del Sur (UNS), San Juan 670, Bahía Blanca, Argentina. \\ ddrittanti@gmail.com \\ *Autor de correspondencia: ncanale@unrn.edu.ar
}

\begin{abstract}
RESUMEN. El análisis sedimentológico e icnológico de la Formación Lajas (Jurásico Medio) en el área de Bajada de Los Molles permitió reconocer depósitos de offshore superior-shoreface inferior que pasan en transición hacia sucesiones de prodelta, frente deltaico y bahía interdistributaria. La presencia de un gran número de depósitos de canales distributarios con abundante contenido de materia orgánica particulada y la muy baja diversidad y abundancia de trazas fósiles, permiten clasificar a este sistema como un delta de tipo fluvio-dominado. En el tramo basal y superior de la sucesión analizada son comunes los depósitos generados por descargas de densidad hiperpícnicas. Internamente las hiperpicnitas conforman sistemas de canal-albardón y canales distributarios, integrados por areniscas masivas y estructuras de deformación por carga, o por un pasaje transicional y recurrente de estructuras sedimentarias tractivas con abundante presencia de materia orgánica particulada en sus caras de avalancha. La mayor diversidad y abundancia de trazas fósiles se reconoce en los depósitos de offshore superior-shoreface inferior, con desarrollo de las icnofacies de Skolithos y Cruziana. Los depósitos deltaicos muestran un contenido de trazas fósiles con menos abundancia y diversidad respecto de los anteriores, mientras que las hiperpicnitas son los depósitos que muestran la menor diversidad y abundancia de trazas fósiles o la ausencia de estructuras biogénicas, situación que reflejaría la condición de mayor estrés dentro del sistema analizado.
\end{abstract}

Palabras clave: Cuenca de retroarco, Delta fluvio-dominado, Trazas fósiles, Hiperpicnitas.

\begin{abstract}
Sedimentology and ichnology of a river-dominated delta, Lajas Formation (Middle Jurassic), Neuquén basin, Argentina. Sedimentologic and ichnologic analysis of the Middle Jurassic Lajas Formation in Bajada de Los Molles area allow recognizing upper offshore-lower shoreface deposits in transition to prodelta, delta front and interdistributary bay succesions. This system is classified as a river-dominated delta due to the presence of distributary channel deposits with huge amounts of particulate organic matter and low diversity and abundance of trace fossils. Hyperpycnites are common in the basal and upper part of the studied section, and form channel-levee and distributarychannel systems, integrated by massive sandstones and load deformation structures, or transitional and recurrent passages of tractive sedimentary structures with abundant particulate organic matter on the foresets. The greatest diversity and abundance of trace fossils are recognized in the upper offshore-lower shoreface deposits where Skolithos and Cruziana ichnofacies occur. Deltaic deposits show trace fossil associations with lower diversity and abundance than the fully marine ones, whereas the hyperpycnite deposits are either unbioturbated or show the lowest diversity and abundance of trace fossils, reflecting the most stressed conditions within the system.
\end{abstract}




\section{Introducción}

El estudio de la sedimentología e icnología en sistemas deltaicos ha tenido gran desarrollo en los últimos años (Coates y MacEachern, 1999, 2000; Bann y Fielding, 2004; Gani et al., 2004; MacEachern et al., 2005; Tonkin, 2012). Los deltas constituyen sistemas sumamente dinámicos en los que las interrelaciones entre los procesos fluviales, de oleaje y de marea varían a través del tiempo y el espacio (Bhattacharya y Giosan, 2003; Bhattacharya, 2006). $\mathrm{Si}$ bien existe un consenso en que la clasificación de un delta se realiza teniendo en cuenta su morfología, costas lobadas para deltas fluvio-dominados, costas rectas para deltas dominados por oleaje, y costas en embudo para deltas afectados por mareas (Galloway, 1975; Boyd et al., 1992), se necesita de un análisis sedimentológico e icnológico integral para establecer claramente la influencia de estos procesos, y cómo varían los mismos. Olariu y Bhattacharya (2006) propusieron que la cantidad de canales distributarios terminales que desarrolla un delta permite establecer cuál es el proceso que domina su construcción y evolución. Estos autores indican que los deltas fluvio-dominados son los que presentan la mayor cantidad de canales distributarios terminales, en contraposición con los deltas dominados por oleaje, que son los que desarrollan la menor cantidad de estos canales. Otro aspecto de interés es considerar la recurrencia que presentan las hiperpicnitas en los depósitos deltaicos. Si bien factores como el clima, la tectónica y el nivel relativo del mar son claves para el desarrollo de estos flujos (Mulder et al., 2003; Plink-Bjorklund y Steel, 2004; Canale et al., 2015), la morfología desarrollada por el sistema deltaico controla significativamente la recurrencia y magnitud de estos procesos. Es por esto que los deltas con mayor posibilidad de preservar hiperpicnitas son los de tipo fluvio-dominados, debido a la gran cantidad de canales distributarios terminales que presentan. Una hiperpicnita muestra recurrencia y pasajes transicionales, tanto verticales como laterales, de estructuras sedimentarias sin límites físicos definidos, rangos texturales variables, gradación inversa-normal, múltiples superficies de reactivación interna y en algunos casos abundante contenido de materia orgánica (Zavala et al., 2001; Mulder et al., 2003; Zavala et al., 2006; Ponce y Carmona, 2011a, b; Ponce et al., 2012; Canale et al., 2015). El estudio de las trazas fósiles asociadas a estos depósitos se ha incrementado de manera considerable en los últimos años (Gani et al., 2004; Ponce et al., 2007; Bhattacharya y MacEachern, 2009; Olivero et al., 2010; Buatois et al., 2011; Carmona y Ponce, 2011; Dasgupta y Buatois, 2012; Canale et al., 2013, 2016; Dasgupta et al., 2016; Lokho et al., 2017), permitiendo inferir las variaciones físico-químicas que generan estos sistemas en la columna de agua y en el sustrato, y cómo estas afectan la distribución temporo-espacial de los organismos.

Los depósitos jurásicos de la Formación Lajas han sido tradicionalmente descriptos como deltas dominados por marea (Gulisano y Pando, 1981; Legarreta y Gulisano, 1989; Leanza et al., 2001; Zavala y González, 2001; McIlroy et al., 2005). En particular, en el área de Bajada de Los Molles, Zavala (1993) reconoció en la sección basal depósitos de frente deltaico integrados por barras de desembocadura deltaica fluvio-dominadas, y depósitos de plataforma, depósitos de estuarios y barras mareales para el intervalo medio. McIlroy et al. (2005) y McIlroy (2007) han interpretado los depósitos de esta unidad como correspondientes a un sistema deltaico macromareal; Gugliotta et al. (2015) asignaron la sección superior de la unidad a subdeltas de derrame con influencia mareal, y Rossi y Steel (2016) definieron un delta arenoso de energía mixta. En esta contribución se presentan y discuten las evidencias sedimentológicas e icnológicas que permiten reinterpretar los depósitos de la Formación Lajas expuestos en la localidad de Bajada de Los Molles como correspondientes a un delta fluvio-dominado reelaborado por descargas hiperpícnicas.

\section{Marco Geológico}

La cuenca Neuquina evolucionó en el lado este de los Andes en Argentina y en la porción central de Chile, entre los $32^{\circ}$ y los $40^{\circ}$ de latitud Sur, limitando al noreste con el Macizo de la Sierra Pintada y al sureste con el Macizo Norpatagónico (Digregorio et al., 1984) (Fig. 1A). La cuenca tiene una forma general triangular y abarca un área de más de $200.000 \mathrm{~km}^{2}$ (Yrigoyen, 1991). Su desarrollo está caracterizado por tres estadios evolutivos: una etapa inicial de rift, comprendida entre el Triásico Tardío y el Jurásico Temprano, una etapa de sag relacionada a subsidencia termal, desarrollada entre el Jurásico Temprano y el Cretácico Temprano; y por último, 
una etapa de antepaís comprendida entre el Cretácico Temprano y el Cenozoico Temprano (Uliana et al., 1989; Franzese y Spalletti, 2001; Howell et al., 2005; Spalletti et al., 2005; Pángaro et al., 2009, entre otros). El registro sedimentario de la cuenca alcanza los $7.000 \mathrm{~m}$ de espesor (Uliana y Legarreta, 1993; Vergani et al., 1995) (Fig. 1A).

La extensa fase de subsidencia termal y extensión regional de trasarco favoreció el desarrollo de ciclos transgresivos-regresivos y la acumulación de sucesiones de rocas sedimentarias de gran espesor y distribución areal (Digregorio, 1978; Gulisano et al., 1984; Legarreta y Gulisano, 1989). Bajo estas condiciones se acumularon las sedimentitas que integran los grupos Cuyo, Lotena y Mendoza, los que se vieron afectados por cambios en las tasas de subsidencia, alzamientos localizados y oscilaciones eustáticas (Bracaccini, 1970; Carbone, 1988; Howell et al., 2005, entre otros). Durante este intervalo también se registra la formación de discordancias angulares en la zona de la Dorsal de Huincul (Orchuela et al., 1981; Ploszkiewicz et al., 1984; Freije et al., 2002). Si bien diversos autores propusieron la reactivación de la dorsal a fines del Jurásico y Cretácico (Ploszkiewicz et al., 1984; Vergani et al., 1995), estudios más recientes demuestran que esta deformación habría sido más extendida y compleja. En particular Freije et al. (2002) reconocieron para el flanco sur del Arroyo Picún Leufú un sistema de discordancias progresivas activo durante el Jurásico Medio-Tardío. Estos autores infieren que el control tectónico en las unidades comprendidas entre las edades Bajociano temprano a Tithoniano tardío habría cumplido un rol principal en la estratigrafía de este intervalo, en comparación con la influencia del clima y las variaciones eustáticas.

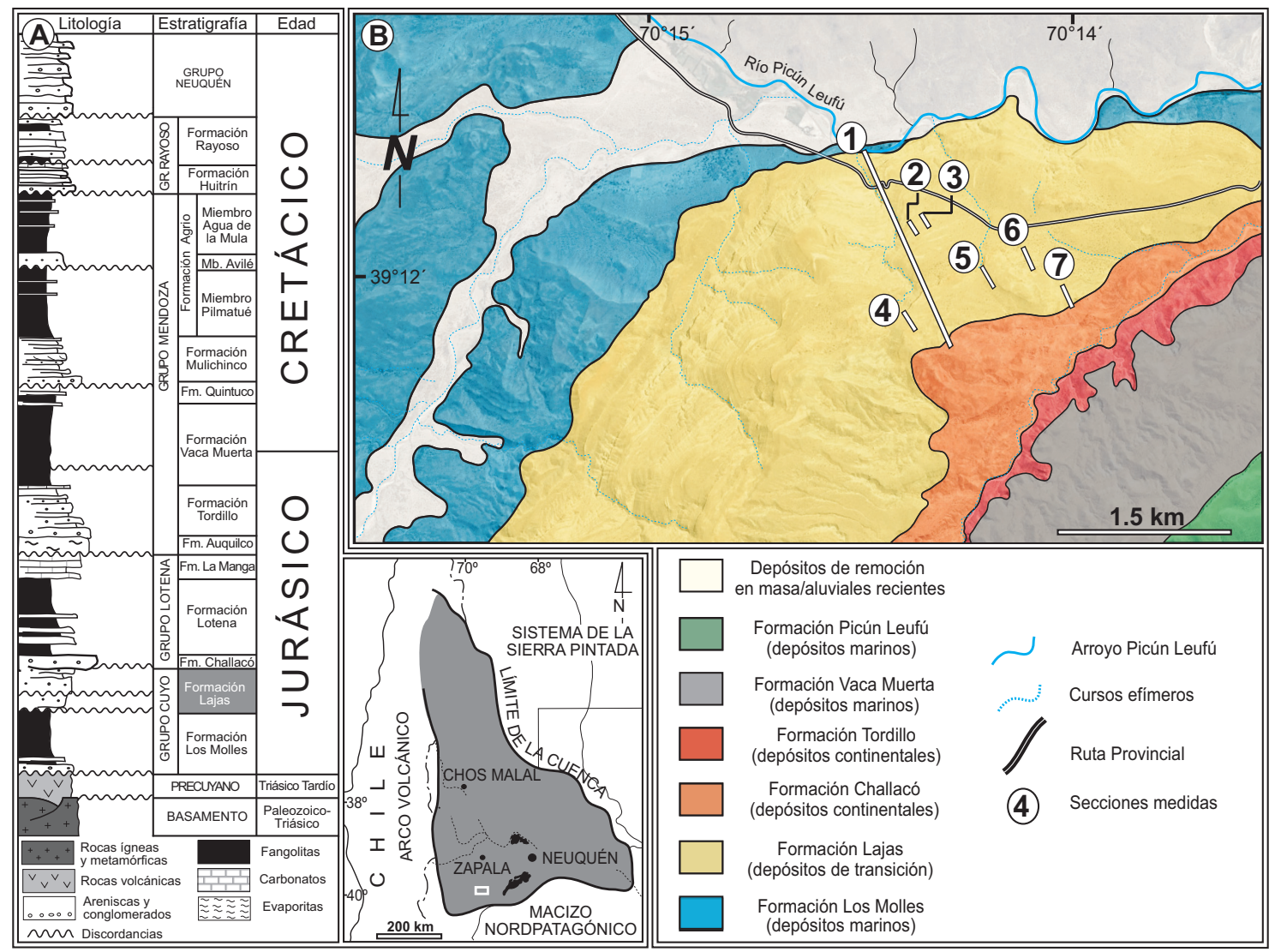

FIG. 1. A. Columna estratigráfica indicando la ubicación de la Formación Lajas (recuadro gris) y mapa de la cuenca Neuquina mostrando la ubicación del área de estudio (recuadro blanco). Modificado de Zavala y González (2001). B. Mapa geológico detallado del área de Bajada de Los Molles con la ubicación de las secciones medidas. 
En el área de Picún Leufú, el Grupo Cuyo está representado por sucesiones dominantemente pelíticas de offshore-shoreface que intercalan con cuerpos lenticulares de areniscas generadas por corrientes de densidad correspondientes a la Formación Los Molles (Dellapé et al., 1979; Gulisano y Gutiérrez Pleimling, 1995; Paim et al., 2008). Esta unidad es sucedida por depósitos deltaicos de la Formación Lajas (Weaver, 1931), los cuales son cubiertos por depósitos aluviales a fluviales de la Formación Challacó (de Ferraríis, 1947; Veiga, 2000) (Fig. 1B). En esta localidad, las rocas sedimentarias de la Formación Lajas han sido originalmente interpretadas como correspondiente a depósitos mareales (Dean, 1987; Gulisano y Pando, 1981; Legarreta y Gulisano, 1989; Rosenfeld y Volkheimer, 1979; Leanza et al., 2001), mientras que en estudios más recientes se han identificado depósitos de plataforma, depósitos deltaicos fluvio-dominados, dominados por oleaje y dominados por marea, además de estuarios y barras mareales (Zavala, 1993; McIlroy et al., 2005; McIlroy, 2007; Gugliotta et al., 2015; Rossi y Steel, 2016).

\section{Asociaciones de facies, contenido icnológico y ambiente depositacional}

La buena exposición que presentan las rocas del Grupo Cuyo en la localidad de Bajada de Los Molles permitió la medición de una sección de detalle completa de la Formación Lajas, con un total de $560 \mathrm{~m}$ de espesor, la cual incluye el contacto con la unidad infrayacente (Formación Los Molles), y con la unidad suprayacente (Formación Challacó), y seis secciones parciales para complementar la interpretación paleoambiental del sistema, la arquitectura del frente deltaico, y la variación temporo-espacial de las asociaciones de facies (Fig. 1B). En esta localidad, la Formación Lajas muestra un arreglo basal progradante que, hacia la parte media y superior, se vuelve agradante (Fig. 2). Estos depósitos están mayormente integrados por areniscas y fangolitas en las que se reconocieron doce facies sedimentarias, cuyas principales características se detallan en la tabla 1.

\subsection{Asociaciones de facies}

Sobre la base de un detallado análisis de la distribución temporo-espacial que muestran las doce facies sedimentarias reconocidas, y teniendo en cuenta la geometría de los estratos que las contienen, se pudieron reconocer seis asociaciones de facies (AF1 a AF6). En cada asociación de facies se establece el índice de bioturbación (BI, por sus siglas en inglés) definido por Taylor y Goldring (1993).

\subsubsection{AF1. Offshore}

Esta asociación de facies está integrada por niveles de fangolitas y areniscas muy finas con materia orgánica particulada, que muestran su fábrica primaria completamente obliterada por bioturbación (BI 5-6). Los estratos presentan geometría tabular y gran continuidad lateral (Figs. 2 y 3A). De manera subordinada se reconocieron niveles de hasta $4 \mathrm{~cm}$ de espesor integrados por pelitas masivas $(\mathrm{Mm})$ o con laminación (Ml), y areniscas con óndulas simétricas (Srw) con presencia de materia orgánica particulada (Fig. 2, Tabla 1). El contenido icnológico se encuentra principalmente representado por Chondrites isp., Curvolithus simplex, Nereites isp., Planolites isp., Protovirgularia isp., Teichichnus isp. y Thalassinoides suevicus en los intervalos más fangosos (Fig. 3B-C), mientras que Skolithos linearis es el icnogénero más recurrente en los niveles de areniscas con óndulas simétricas.

Interpretación. Las características sedimentológicas y el contenido icnológico que presentan los distintos estratos que integran la AF1 permiten reconocer que los procesos de decantación habrían predominado sobre los procesos tractivos. La baja tasa de sedimentación favorece así la obliteración completa de las estructuras primarias por efecto de la bioturbación (Fig. 3A-C). La icnofauna muestra una moderada a alta diversidad y abundancia, sugiriendo condiciones marinas relativamente normales con desarrollo de una icnofacies de Cruziana. La presencia de delgados niveles de areniscas finas con óndulas de oleaje se asigna a eventos de tormenta, en los que se reconoce la presencia de algunos componentes de la icnofacies de Skolithos. La materia orgánica presente en esta asociación de facies habría sido aportada, conjuntamente con el fango, por decantación desde una pluma hipopícnica asociada a un sistema fluvial cercano.

\subsubsection{AF2. Shoreface}

Se encuentra conformada por areniscas finas a medias, dispuestas en bancos de hasta $70 \mathrm{~cm}$ de espesor que conforman cuerpos de geometría tabular con gran extensión regional. Internamente muestran 


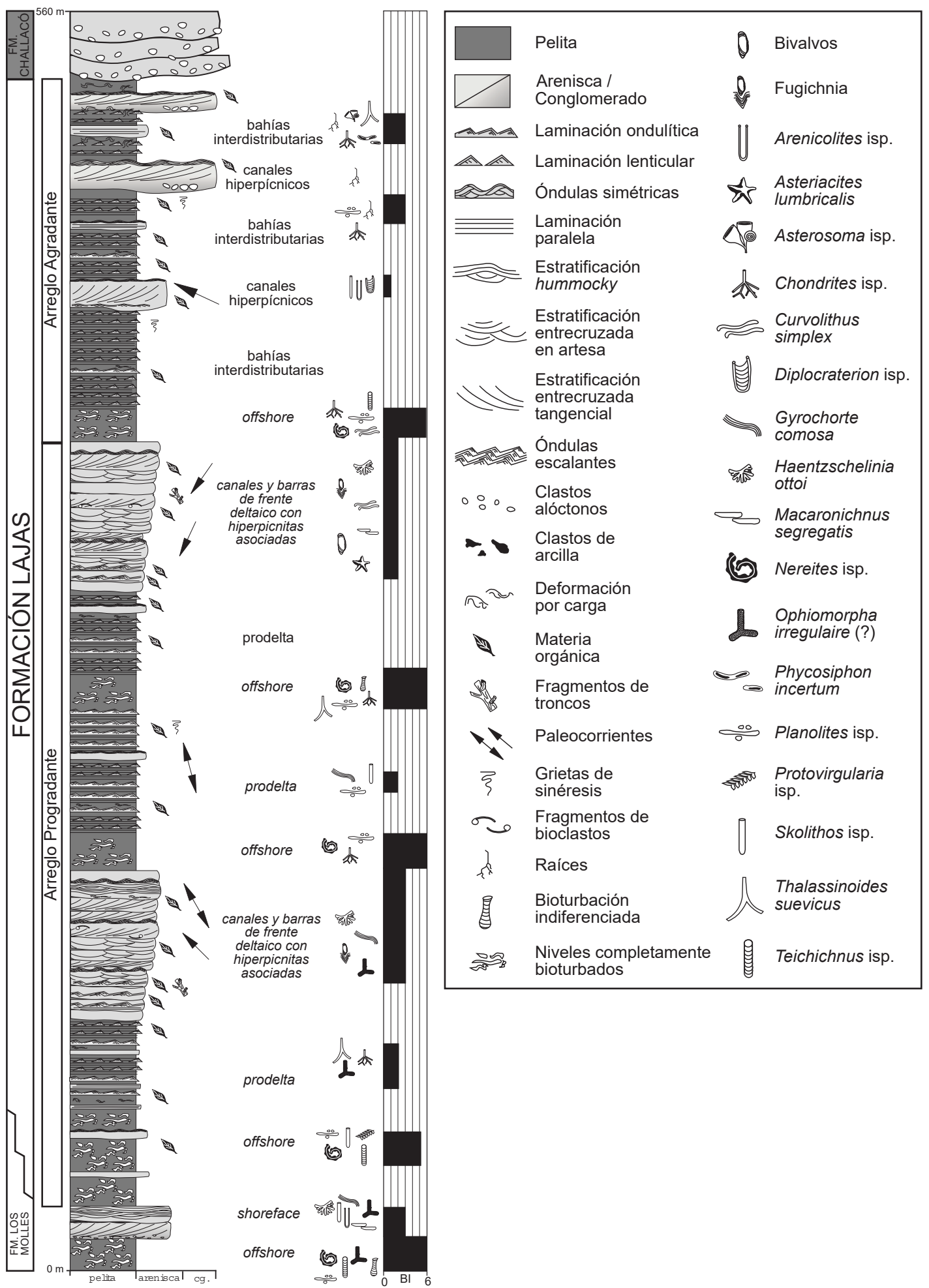

FIG. 2. Sección esquemática de la Formación Lajas en la localidad de Bajada de Los Molles, donde se indican los ambientes sedimentarios y el índice de bitourbación (BI por sus siglas en inglés). cg.: conglomerado. 


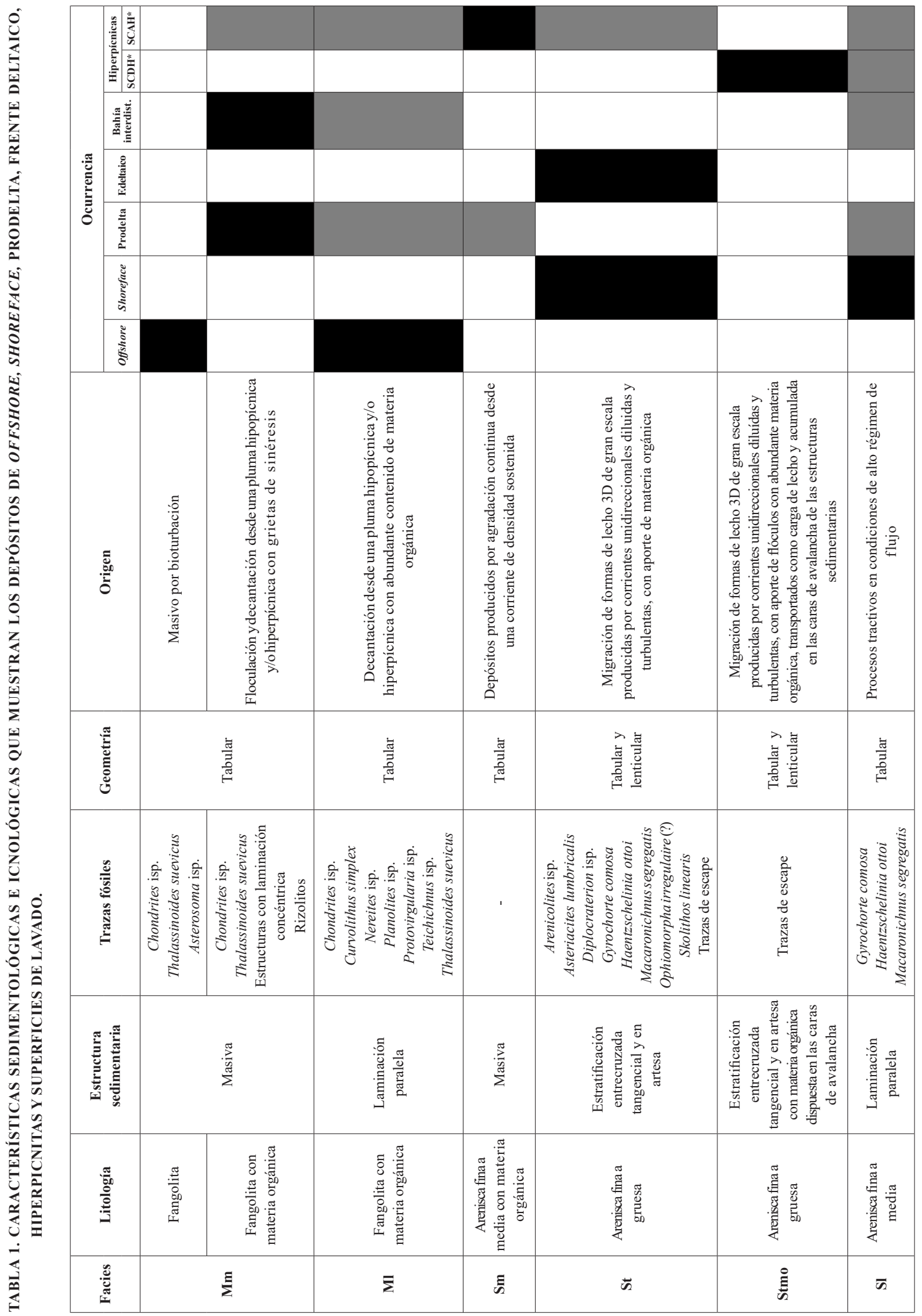




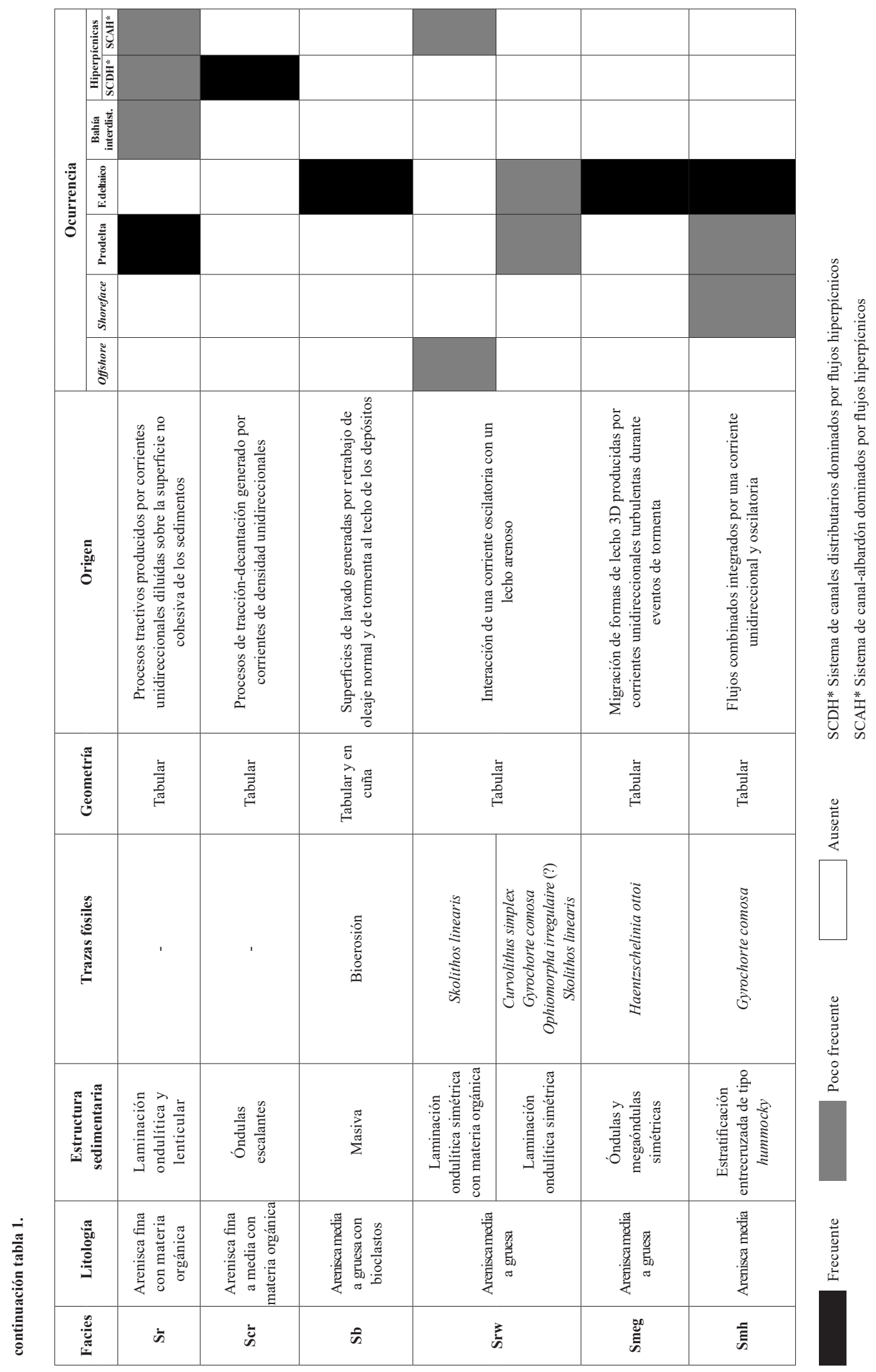



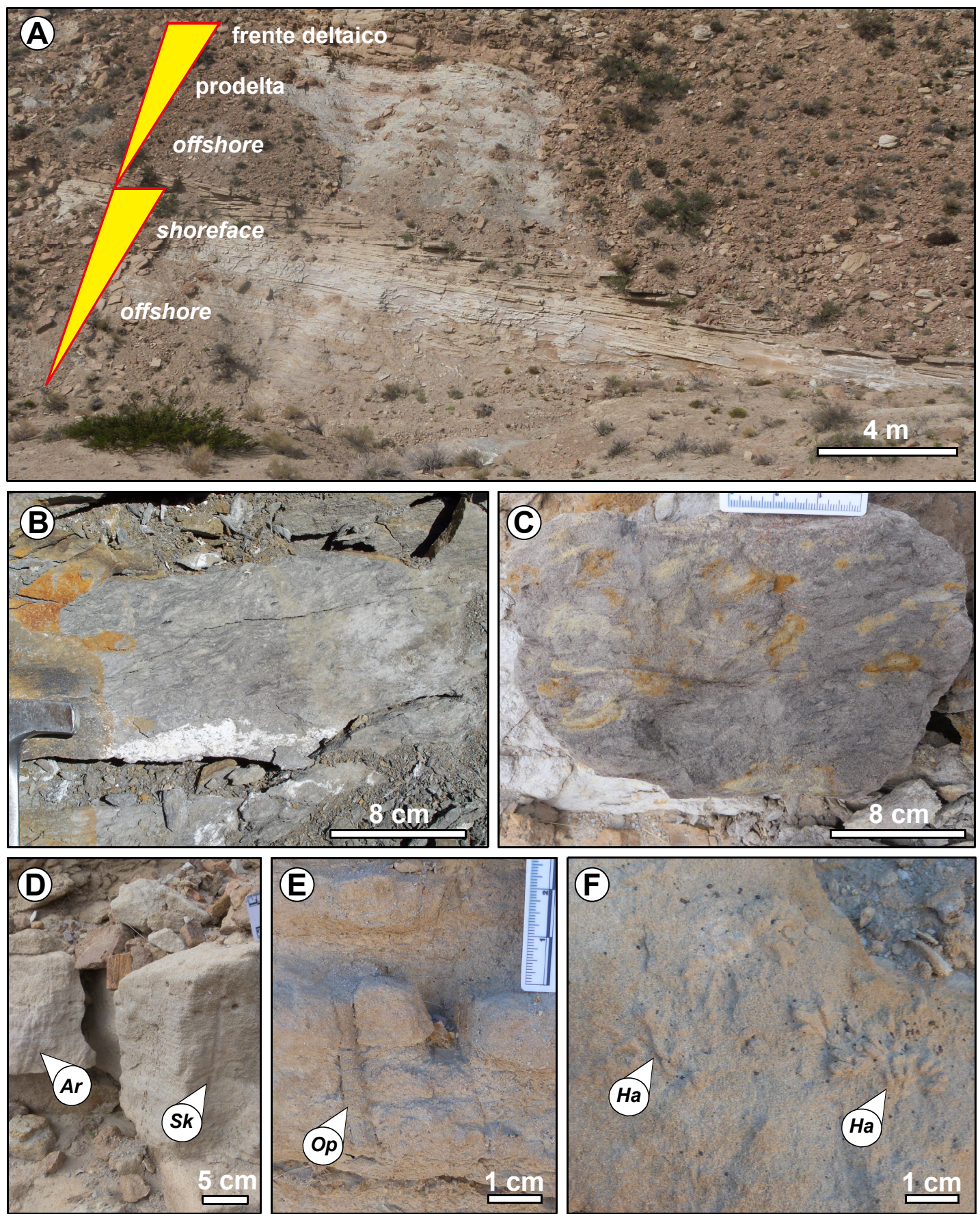

FIG. 3. A. Vista panorámica que muestra una sucesión grano-creciente interpretada como un pasaje entre depósitos de offshore (AF1)shoreface (AF2). Este arreglo es sucedido por otro grano creciente integrado por depósitos de offshore (AF1)-prodelta (AF3) y frente deltaico (AF4). Las barras amarillas indican la tendencia granulométrica de los depósitos. B-C. Vista en sección de niveles completamente obliterados por bioturbación correspondientes a la asociación de facies de offshore (AF1). D-E. Vista en sección de niveles de areniscas correspondientes a las asociaciones de facies de shoreface (AF2) donde se observan ejemplares de Arenicolites isp. (Ar), Skolithos linearis (Sk) y Ophiomorpha irregulaire (?) (Op). F. Vista en planta de ejemplares de Haentzschelinia ottoi (Ha) en los depósitos arenosos de shoreface (AF2). 
laminación paralela $(\mathrm{Sl})$, estratificación entrecruzada tangencial de bajo ángulo (St) y estratificación entrecruzada de tipo hummocky (Smh) (Figs. 2, 3 A y Tabla 1). Presentan escasos clastos alóctonos y bioclastos asociados, y no se observó la presencia de materia orgánica. El contenido icnológico, con un BI de 2-3, comprende estructuras biogénicas asignadas a Skolithos linearis, Arenicolites isp., Haentzschelinia ottoi y Ophiomorpha irregulaire (?) al techo de los paquetes (Fig. 3D-F). Macaronichnus segregatis se encuentra presente en los niveles que muestran estratificación entrecruzada de muy bajo ángulo, y Gyrochorte comosa al techo de algunos bancos con estratificación entrecruzada de tipo hummocky (Smh). La AF2 se reconoce solamente a la base de la sucesión analizada, y no supera los $5 \mathrm{~m}$ de espesor (Figs. 2 y 3 ).

Interpretación. La AF2 representa depósitos acumulados en zonas ubicadas por encima del nivel de oleaje de buen tiempo. Las areniscas se habrían acumulado en sistemas de barras y dunas 2D-3D producidas por la acción de flujos unidireccionales, mientras que la presencia de estructuras de tipo hummocky se vincula al desarrollo de corrientes unidireccionales y oscilatorias (Harms et al., 1975; Swift et al., 1986; Arnott y Southard, 1990). La ausencia de concentraciones significativas de materia orgánica en esta asociación de facies sugiere un ambiente de acumulación alejado de un área de aporte fluvial directo, con acción de oleaje como proceso dominante. El contenido icnológico muestra una diversidad moderada con desarrollo de una icnofacies de Skolithos. La presencia de Macaronichnus segregatis asociada a depósitos con estratificación tangencial de bajo ángulo sugiere que parte de esta asociación de facies podría representar posiciones de foreshore (Clifton y Thompson, 1978; Pemberton et al., 2001). Asimismo, la presencia de Gyrochorte comosa en los niveles con estratificación entrecruzada de tipo hummocky se interpreta como la colonización de los depósitos de tormenta por parte de organismos oportunistas.

\subsubsection{AF3. Prodelta}

Esta asociación de facies está compuesta por una alternancia de capas centimétrica de pelitas y areniscas muy finas, que en conjunto conforman arreglos heterolíticos (Figs. 2 y 4A-C y Tabla 1). Los niveles de areniscas finas son masivos (Sm) o muestran laminación paralela $(\mathrm{Sl})$, ondulítica y lenticular ( $\mathrm{Sr}$ ), mientras que las pelitas pueden ser masivas (Mm) o laminadas (Ml), y muestran comúnmente estructuras de deformación por carga. El contenido de materia orgánica particulada es muy abundante, así como el desarrollo de grietas de sinéresis, además de la presencia de delgados niveles de areniscas medias a finas con ondulitas simétricas (Srw) y estratificación entrecruzada de tipo hummocky (Smh) de pequeña escala. El contenido icnológico es escaso (BI 1-2) y está representado por ejemplares de Chondrites isp., Planolites isp. $\mathrm{y}$ Thalassinoides suevicus en los arreglos heterolíticos (Fig. 4D-E), y ejemplares de Gyrochorte comosa, Ophiomorpha irregulaire (?) y Skolithos linearis en los paquetes con ondulitas simétricas. Estos estratos presentan geometrías tabulares, gran extensión lateral y se diferencian de la AF1 por la buena preservación de las estructuras sedimentarias primarias, el alto contenido de materia orgánica y la baja diversidad y abundancia de trazas fósiles (Fig. 4).

Interpretación. La AF3 se habría acumulado por la alternancia entre procesos de tracción y decantación. La presencia de fangolitas masivas con abundante contenido de fitodetrito indica que los procesos de floculación habrían sido muy importantes, generando depósitos de fango fluido de espesor variable (Fig. 4B). Estos niveles no presentan trazas fósiles asociadas, lo que puede relacionarse a problemas de turbidez y pobre oxigenación del sustrato, sumado a la mala preservación de estructuras biogénicas en sustratos soposos (MacEachern et al., 2005). La presencia recurrente de grietas de sinéresis indicaría cambios de salinidad producidos por el aporte de agua dulce desde una pluma hipopícnica o hiperpícnica, vinculada a una descarga fluvial cercana (ver también interpretaciones alternativas de Plummer y Gostin, 1981). El contenido icnológico de los depósitos heterolíticos representa una icnofacies de Cruziana empobrecida, principalmente relacionada con problemas de salinidad y turbidez (MacEachern et al., 2005; Buatois y Mángano, 2011; Vieira de Luca y Basilici, 2013). La presencia recurrente de intervalos de areniscas con óndulas simétricas y estratificación entrecruzada de tipo hummocky de pequeña escala, indica que los procesos de retrabajo por oleaje normal y de tormenta fueron frecuentes, favoreciendo el establecimiento de organismos suspensívoros correspondientes a una icnofacies de Skolithos empobrecida. 

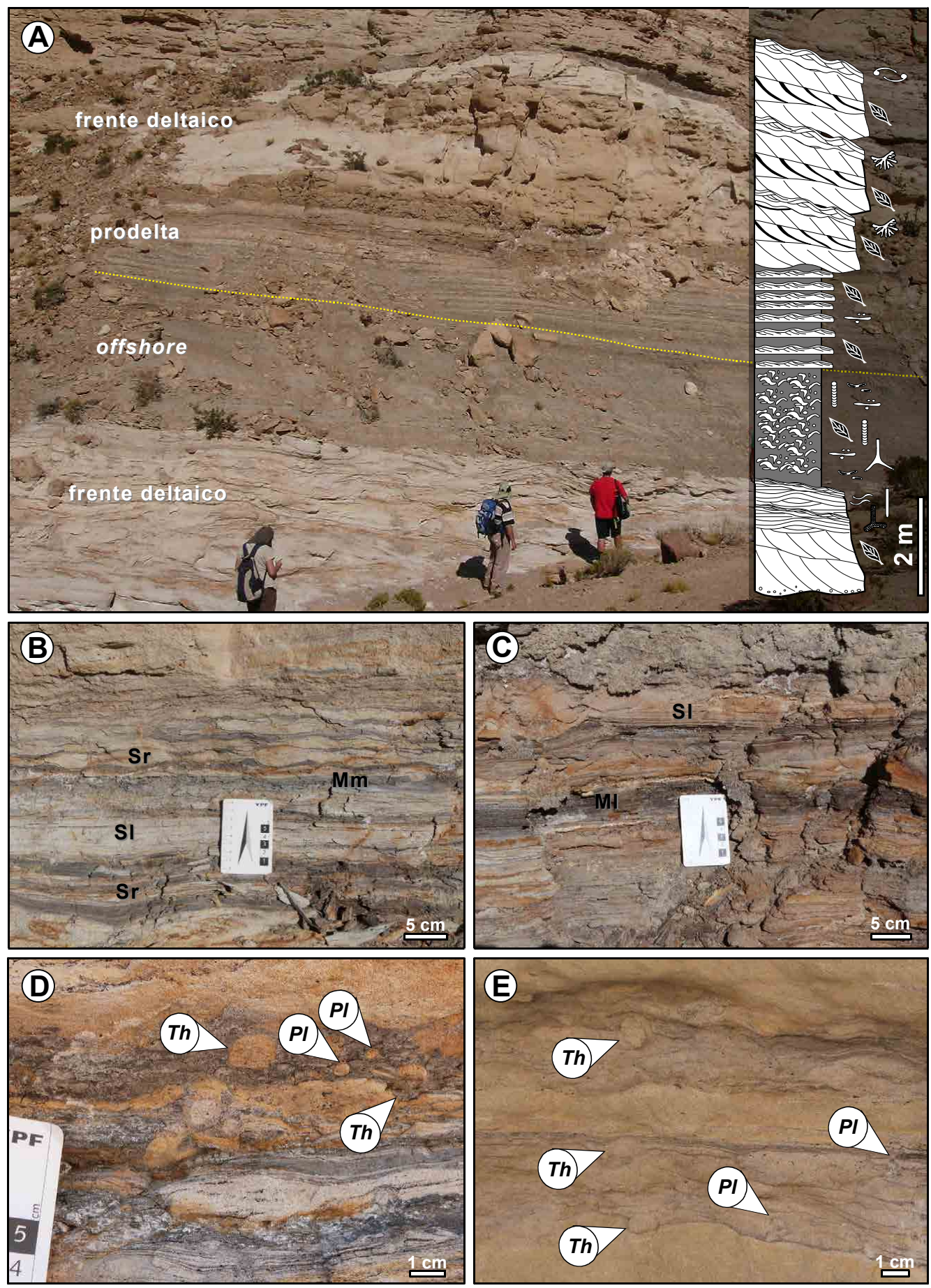

FIG. 4. A. Vista panorámica y sección esquemática mostrando las transiciones observadas entre ambientes de offshore (AF1), prodelta (AF3) y frente deltaico (AF4). Note el aspecto masivo generado por la bioturbación en los depósitos de offshore (AF1) y la preservación de la laminación en los depósitos de prodelta (AF3). B-C. Niveles de heterolitas de prodelta (AF3) integrados por la alternancia entre niveles de areniscas con laminación paralela ( $\mathrm{Sl}$ ) y ondulítica ( $\mathrm{Sr}$ ) que muestran abundante materia orgánica, y de fangolitas masivas (Mm) y laminadas (M1). D-E. Facies de prodelta (AF3) que muestran estructuras biogénicas asignadas a Planolites isp. $(P l)$ y Thalassinoides $(T h)$. 


\subsubsection{AF4. Frente Deltaico}

Esta asociación de facies se encuentra integrada por areniscas finas a gruesas que incluyen concentraciones variables de materia orgánica particulada y fragmentos de troncos, así como fragmentos de bioclastos (Fig. 2 y Tabla 1). Los estratos presentan geometría lenticular en sección transversal al flujo (Fig. 5A), y sigmoidal en sección longitudinal al flujo (Fig. 5B). Internamente estos depósitos muestran dos arreglos bien diferenciados. El primer arreglo está integrado por areniscas con estratificación entrecruzada tangencial y en artesa de gran escala (St) (Fig. 6A), que puede mostrar estructuras de deformación por carga, $\mathrm{y}$ hacia el techo ondulitas simétricas (Srw) y estratificación entrecruzada de tipo hummocky (Smh). Estos depósitos presentan materia orgánica particulada y fragmentos de troncos, y alcanzan espesores de hasta $15 \mathrm{~m}$, con sets que varían entre $0,2 \mathrm{~m}$ y $2 \mathrm{~m}$. El contenido icnológico es moderado (BI: 3), y se encuentra integrado por Asteriacites lumbricalis, Curvolithus simplex, Gyrochorte comosa, Haentzschelinia ottoi, Macaronichnus segregatis, Ophiomorpha irregulaire (?) y trazas de escape -fugichnia-, (Fig. 6B-E). El segundo arreglo, muestra areniscas con estratificación entrecruzada de tipo hummocky (Smh), óndulas (Srw) y megaóndulas simétricas (Smeg) y estructuras de deformación por carga (Fig. 6F). Estos depósitos también presentan materia orgánica particulada y troncos, mientras que su contenido icnológico se caracteriza por la presencia de Haentzschelinia ottoi como estructura biogénica dominante (Fig. 6G-H). Los espesores reconocidos para estos estratos alcanzan los $5 \mathrm{~m}$, y se encuentran integrados por sets con potencias que varían entre $0,2 \mathrm{~m}$ y $2 \mathrm{~m}$.

$\mathrm{Al}$ techo de ambos arreglos es común la presencia de capas con geometría tabular y en cuña con bases netas-erosivas de hasta $80 \mathrm{~cm}$ de espesor y centenares de metros de extensión lateral (flechas blancas en Fig. 5B). Internamente, estos cuerpos se encuentran integrados por areniscas calcáreas masivas con abundante contenido de bioclastos (Figs. 2, 6I-J y Sb en Tabla 1), en especial restos de bivalvos trigónidos y ostreidos con evidencias de bioerosión. En algunos casos se reconoció la presencia de trigónidos en posición de vida (flecha blanca en Fig. 6J).

Interpretación. En general la AF4 representa la migración de sistemas de barras 3D dentro de canales distributarios terminales de pequeña escala (100 $\mathrm{m}$ de ancho y $5 \mathrm{~m}$ de profundidad). La presencia de materia orgánica particulada y troncos refleja el aporte de sistemas fluviales activos, ya sea como carga de lecho o desde una pluma hipopícnica. La ausencia de estructuras biogénicas de organismos suspensívoros indicaría que las condiciones de turbidez fueron importantes, mientras que la presencia de organismos oportunistas (e.g., Gyrochorte comosa) colonizando el tope de las barras afectadas por acción de oleaje, marca el establecimiento de ventanas de colonización entre las sucesivas etapas de construcción de las mismas o durante su abandono (Fig. 6C). Las barras que muestran estructuras sedimentarias de óndulas, megaóndulas simétricas y estratificación entrecruzada de tipo hummocky reflejan que los procesos de flujos oscilatorios producidos por acción de oleaje normal y de tormenta fueron dominantes, causando la reelaboración completa de los depósitos primarios (Fig. 6F-G). Estas barras son las que exhiben las ventanas de colonización temporalmente más extendidas. La presencia recurrente de cuerpos tabulares de areniscas calcáreas reelaborando el techo de las asociaciones de barras deltaicas se interpretan como "superficies de lavado" (ver Canale et al., 2015). Estas superficies se producen por la erosión y retransporte de las fracciones clásticas más finas y la acumulación progresiva de las fracciones clásticas más gruesas (bioclastos) (Fig. 6I-J). Si bien los arreglos que muestran estos paquetes son similares a los producidos por las superficies de ravinamiento transgresivas, difieren de ellas en que no presentan continuidad regional (flechas blancas en Fig. 5B).

\subsubsection{AF5. Bahía Interdistributaria}

Esta asociación de facies está integrada principalmente por fangolitas masivas $(\mathrm{Mm})$ con estructuras de deformación por carga, y de manera subordinada, por fangolitas laminadas ( $\mathrm{Ml}$ ) y areniscas finas con laminación paralela $(\mathrm{Sl})$ y ondulítica $(\mathrm{Sr})$, conformando depósitos de estratificación mixta (Fig. 7 y Tabla 1). Estos depósitos tienen gran extensión regional y geometría tabular. Los diques clásticos conectando estratos arenosos son comunes, así como las grietas de sinéresis y los restos de hojas y tallos excelentemente preservados y con sus ejes mayores dispuestos de manera paralela al plano de estratificación (Fig. 7B-C). El contenido icnológico muestra una baja diversidad y abundancia (BI 2-3), y está representado por Thalassinoides suevicus, Chondrites isp., Asterosoma isp., Phycosiphon incertum y trazas de raíces (Fig. 7A y D). Esta asociación de facies ha sido reconocida solamente en el tramo superior de la 

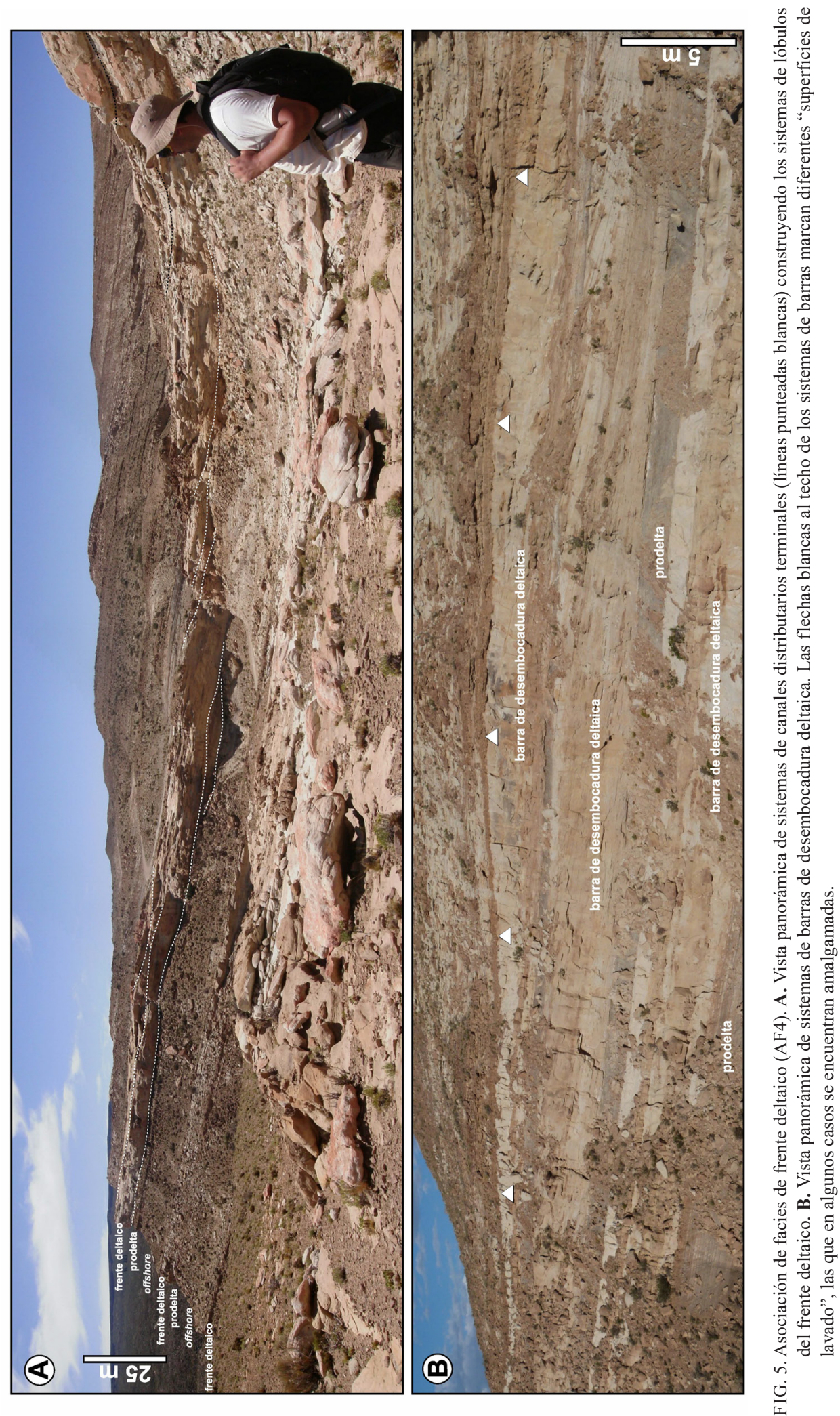

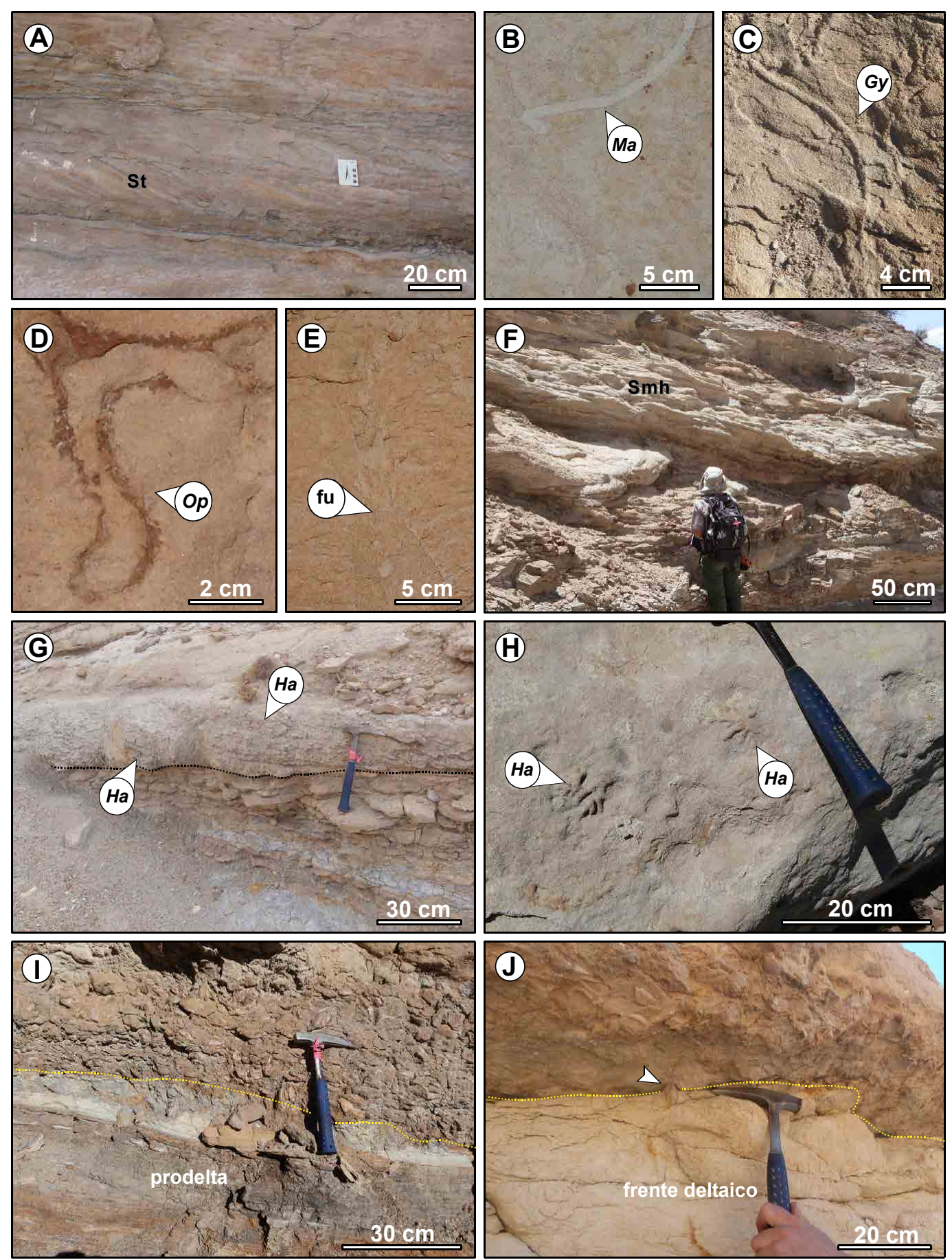

FIG. 6. Asociación de facies de frente deltaico (AF4). A. Barras de desembocadura deltaicas con estratificación entrecruzada tangencial de gran escala (St). B. Vista en planta de Macaronichnus segregatis (Ma). C. Vista en planta de ejemplares Gyrochorte comosa $(G y)$. D. Vista en sección de ejemplares de Ophiomorpha irregulaire (?) (Op). E. Vista en sección de una traza de escape (fu). F. Barras de desembocadura deltaica con óndulas de oleaje y estratificación entrecruzada de tipo hummocky (Smh). G-H. Vista en sección y en planta respectivamente de una barra de frente deltaico donde se reconocen ejemplares de Haentzschelinia ottoi (Ha). I-J. Vista en sección de niveles de bioclastos producidos por eventos de tormentas. En este caso la base es erosiva (líneas punteadas amarillas). En $\mathbf{J}$ note la presencia de bivalvos en posición de vida (flecha blanca). 

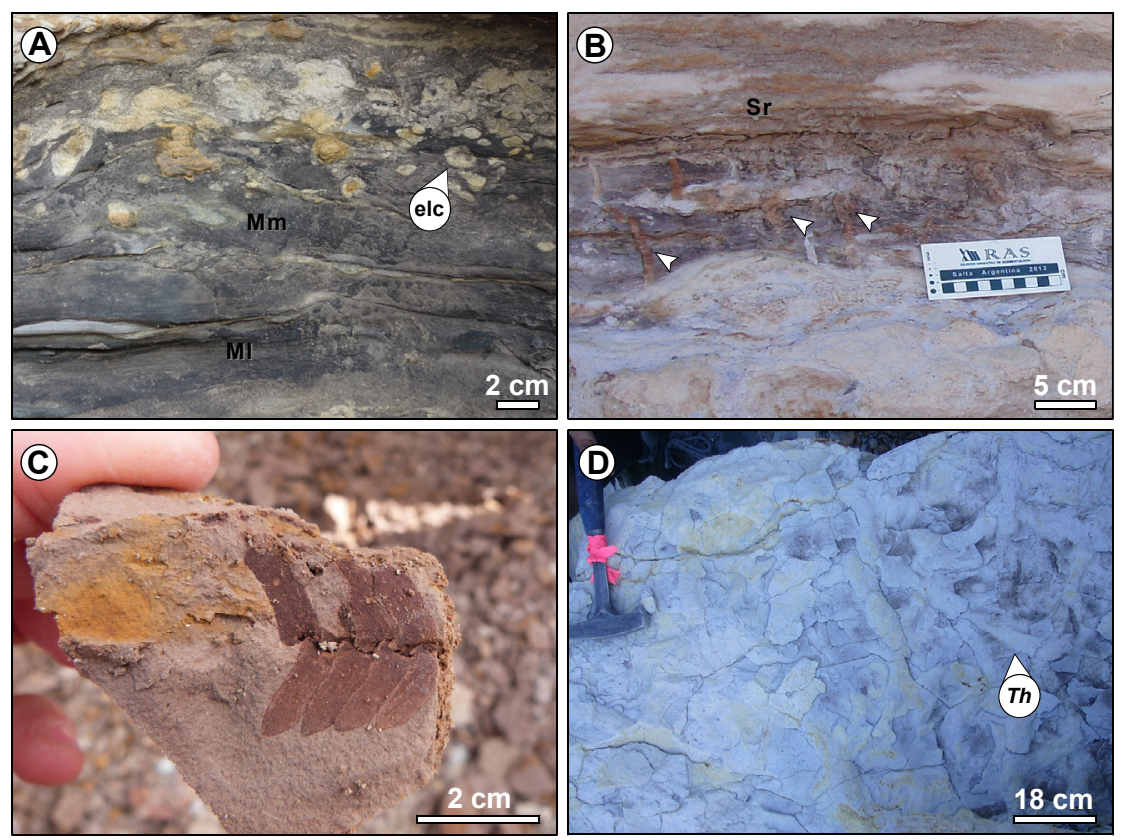

FIG. 7. Asociación de facies de bahía interdistributaria (AF5). A. Vista en sección de fangolitas masivas que hacia el techo muestran estructuras con laminación concéntrica (elc). B. Vista en sección de fangolitas laminadas con diques clásticos (flechas blancas) conectando niveles de areniscas con laminación ondulítica (Sr). C. Vista en planta de niveles de pelitas con impresiones de hojas excelentemente preservadas. D. Vista en planta de niveles de fangolitas masivas con ejemplares de Thalassinoides suevicus (Th).

sección analizada, donde se encuentra parcialmente erosionada por sistemas de canal-albardón dominados por flujos hiperpícnicos de gran escala (Fig. 2).

Interpretación. Esta asociación de facies corresponde a depósitos de planicie fangosa y mixta acumulados en bahías interdistributarias deltaicas. Los niveles de fango fluido, integrados por fangolitas masivas acumuladas por procesos de floculación, son las facies sedimentarias dominantes. La presencia de grietas de sinéresis sugiere que los cambios de salinidad, vinculados con una circulación restringida, habrían sido frecuentes. La presencia de diques clásticos conectando niveles arenosos resultaría de la combinación entre una alta tasa de sedimentación (producida durante descargas de densidad) y la presencia de niveles de fango fluido, los cuales habrían favorecido la licuefacción de las arenas desde los niveles infrayacentes hacia los suprayacentes. La baja diversidad y abundancia de trazas fósiles, sumado a la gran abundancia de restos de hojas y tallos excelentemente preservados en estos ambientes sugieren condiciones disóxicas.

\subsubsection{AF6. Hiperpicnitas}

Esta asociación de facies se encuentra representada por dos arreglos característicos. El primero de estos corresponde a sistemas de canales distributarios dominados por flujos hiperpícnicos de pequeña escala (SCDH en Tabla 1) y el segundo a sistemas de canal-albardón dominados por flujos hiperpícnicos de gran escala (SCAH en Tabla 1).

Los sistemas de canales distributarios dominados por flujos hiperpícnicos de pequeña escala (SCDH), están conformados por depósitos de areniscas medias a gruesas acumuladas en paquetes de hasta $3 \mathrm{~m}$ de espesor, que en general conforman cuerpos lenticulares de 50 a $100 \mathrm{~m}$ de ancho y 3 a $4 \mathrm{~m}$ de espesor. Internamente muestran un pasaje transicional y recurrente entre areniscas con estratificación entrecruzada tangencial y en artesa (Stmo), óndulas escalantes (Scr), óndulas de corriente ( $\mathrm{Sr}$ ) y laminación paralela (Sl) con deformación por carga (Fig. 8A-C). A menudo se reconoce la presencia de agregados integrados por fitodetrito y fango tapizando las caras de avalancha de las diferentes estructuras sedimentarias (Fig. 8A-C, Tabla 1). El contenido icnológico es escaso (BI 1), siendo las trazas de escape las únicas reconocidas (Fig. 8C). Estos paquetes son muy comunes en el tramo inferior de la sección y se encuentran truncando a las asociaciones de facies de frente deltaico y prodelta (Fig. 2). 

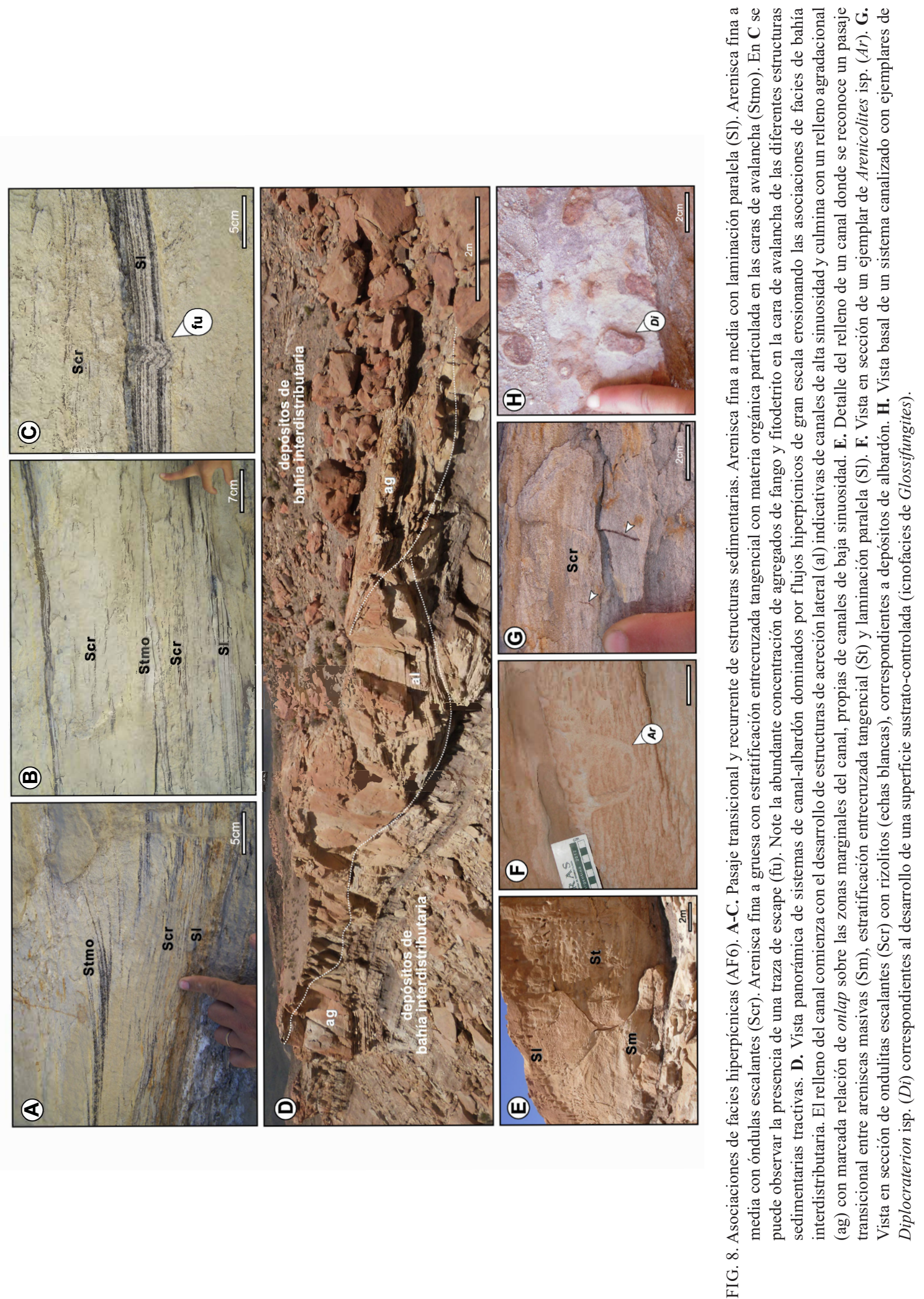
Los sistemas de canal y albardón dominados por flujos hiperpícnicos de gran escala (SCAH), están representados por depósitos de hasta $400 \mathrm{~m}$ de ancho y 7,5 $\mathrm{m}$ de espesor, bases erosivas y geometría lenticular (Fig. 8D). Internamente están compuestos por conglomerados y areniscas finas a gruesas que muestran pasajes transicionales entre estructuras con estratificación entrecruzada tangencial y en artesa $(\mathrm{St})$, laminación paralela (Sl) y masivas (Sm) (Fig. 8E). De manera recurrente, a la base de estos cuerpos se reconocen estructuras de deformación por carga y clastos de arcilla, mientras que hacia el techo pueden presentar ondulitas simétricas (Srw). El relleno de los canales muestra capas con tres tipos de arreglos bien definidos. El primero con una relación de onlap sobre la superficie lenticular, el segundo con sets de capas sigmoidales cuyas superficies inclinan en sentido perpendicular a la dirección del flujo, y el último presenta sets de capas sigmoidales que hacia el techo muestran relaciones de onlap (Fig. 8D) . Los depósitos de albardón asociados a los canales se encuentran integrados por una alternancia centimétrica entre niveles de areniscas con óndulas escalantes (Scr) y laminación ondulítica y lenticular (Sr), y fangolitas laminadas (Ml) o masivas (Mm). Trazas asignadas a Skolithos linearis, Arenicolites isp., estructuras con laminación concéntrica y rizolitos fueron observadas en estos depósitos (BI 1-2) (Fig. 8F-G). También se han observado ejemplares de Diplocraterion isp. con límites netos asociados a las superficies de erosión de los canales (Fig. 8H). Estos arreglos han sido reconocidos solamente en la parte superior de la sección, donde se encuentran truncando a las asociaciones de facies de bahía interdistributaria.

Interpretación. El pasaje transicional y recurrente que muestran las estructuras sedimentarias tractivas que integran estos depósitos se interpreta como variaciones de concentración y velocidad de una corriente de densidad hiperpícnica (Mulder et al., 2003; Zavala et al., 2006; Ponce y Carmona, 2011a, b) (Fig. 8A-B). Bajo estas condiciones se produce la construcción y migración de barras, las que constituyen el relleno de sistemas de canales distributarios dominados por flujos hiperpícnicos de pequeña escala. La abundante presencia de agregados integrados por fitodetrito y fango que tapizan las caras de avalancha de las barras indica que los procesos de floculación han sido muy importantes durante la construcción y migración de estas formas de lecho (Fig. 8A-C). Estos agregados habrían tenido equivalencia hidráulica con las arenas, siendo transportados conjuntamente como carga de lecho y acumulados en las caras de avalancha de estructuras sedimentarias con estratificación entrecruzada tangencial y óndulas escalantes (Tabla 1). Los sistemas de canal-albardón de gran escala representan el relleno de canales hiperpícnicos proximales. El arreglo interno que muestran los canales, con relaciones de onlap, set de capas sigmoidales o la combinación de ambos, indica una alternancia entre canales de baja y alta sinuosidad (Fig. 8D). La relación de corte que muestran con las asociaciones de facies de planicie fangosa y mixta, indican el carácter proximal de los sistemas de canal-albardón de gran escala respecto a los sistemas de canales distributarios de pequeña escala. La presencia de rizolitos en niveles de areniscas finas con óndulas escalantes, interpretados como depósitos de albardón, soportan también el carácter somero de estos sistemas canalizados de gran escala (Fig. 8G). La preservación de niveles de areniscas masivas, de espesores métricos, con estructuras de deformación por carga y la gran dispersión de paleocorrientes que muestra el relleno de los canales, indica una alta tasa de sedimentación y el desarrollo de sistemas fluviales de alta sinuosidad. La presencia de superficies sustrato-controladas con desarrollo de la icnofacies de Glossifungites, con ejemplares de Diplocraterion isp., se relaciona con procesos de avulsión autigénicos y alogénicos, asociados a la migración de canales. La turbidez del agua, los cambios de salinidad producidos por el aporte de agua dulce al sistema y la alta tasa de sedimentación habrían sido los factores de estrés que controlaron el desarrollo de las asociaciones icnológicas en estos intervalos. La presencia de ondulitas simétricas al techo de los canales marca las pausas entre descargas fluviales de magnitud y los momentos en que la acción de oleaje fue un proceso significativo en la reelaboración del depósito.

\section{Discusión}

\subsection{Paleogeografía de la cuenca Neuquina: implicancias en el desarrollo de procesos fluviales, de oleaje y de marea}

Durante el Jurásico y gran parte del Cretácico, la cuenca Neuquina se comportó como una cuenca de retroarco, conectada al proto-Pacífico a través 
de estrechos pasajes entre arcos de islas volcánicos (Vergani, 2005; Mosquera y Ramos, 2006; Berdini et al., 2004; entre otros). La dinámica de la subducción y la evolución del arco volcánico a lo largo del margen oeste de la cuenca favorecieron el desarrollo de etapas alternantes entre conexión episódica y aislamiento del depocentro marino (Uliana y Legarreta, 1993). Más aún, Vicente (2005) propuso que desde el Hettangiano los depósitos del Grupo Cuyo habrían presentado su mayor conexión con el mar abierto al sur de la provincia de Mendoza, en lo que se conoce como el golfo o estrecho de Curepto. Independientemente del número de conexiones con el mar abierto, en este tipo de cuencas marginales, los efectos de la marea son severamente atenuados ya que su desarrollo depende de la sección transversal, profundidad y longitud que tengan las conexiones entre la cuenca y el océano abierto (Jeon et al., 2014). En consecuencia, la amplitud de marea se reduce y disminuye de manera significativa desde estas conexiones hacia el interior de la cuenca, como ocurre actualmente en el Mar de Japón, Mar Negro, Mar Mediterráneo o el Mar Báltico (The Open University, 1999; Jeon et al., 2014; Wetzel, comunicación escrita, 2015). Esta situación provocaría que los depósitos muestren arreglos que resultan propios de aquellos dominados por procesos fluviales y de oleaje, por sobre los de marea (Canale et al., 2015; Ponce et al., 2015). Cabe mencionar que este análisis se contradice con las interpretaciones presentadas en estudios previamente realizados en el área de Bajada de Los Molles por McIlroy et al. (2005) y McIlroy (2007), quienes mencionan una inusual amplificación de la marea en el registro de los depósitos de la Formación Lajas, y también con los estudios realizados por Gugliotta et al. (2015) quienes analizaron los depósitos cuspidales de la Formación Lajas, y propusieron un sistema deltaico fluvio-dominado con influencia micromareal, similar a lo que sucede actualmente en los deltas del Mississippi, Atchafalaya o Wax Lake, todos ellos con un rango micromareal (0,4 m) (Mikhailov y Mikhailova, 2010; Shaw y Mohrig, 2014). Sin embargo, Canale et al. (2013, 2015) y Ponce et al. (2015) sobre la base de estudios realizados en diferentes localidades de la Formación Lajas, analizaron el origen de estructuras sedimentarias asignadas originalmente a procesos de difusión mareal (e.g., tidal bundles), reinterpretándolas como el producto de descargas de densidad fluvio-derivadas, y destacando también la ausencia de estructuras biogénicas (e.g., tidalitas tubulares), diagnósticas de la acción de procesos de difusión mareal (Canale et al., 2015).

\subsection{Arquitectura del frente deltaico}

El análisis arquitectural de los depósitos deltaicos de la Formación Lajas en la localidad de Bajada de Los Molles es muy complejo debido a la interacción que se produce entre la actividad tectónica y las tasas de sedimentación variables. Estos controles (alogénicos y autigénicos) son los responsables de la construcción del frente deltaico donde se reconocen procesos de progradación, agradación y avulsión de sistemas de barras de desembocadura deltaica asociadas a canales distributarios terminales. Olariu y Bhattacharya (2006) definieron los canales distributarios terminales como aquellos que se extienden de manera subácuea hasta la última expresión canalizada dentro del frente deltaico y que se encuentran asociados con el desarrollo de barras de desembocadura deltaica. Los complejos de canales distributarios terminales y barras de desembocadura deltaica de la Formación Lajas muestran espesores de hasta $5 \mathrm{~m}$, y extensiones laterales no superiores a los $400 \mathrm{~m}$, con tres tipos de arreglos internos característicos, denominados I, II y III. El arreglo I muestra sistemas de canales y barras integrados por areniscas medias a finas con estratificación entrecruzada en artesa y tangencial, laminación paralela y ondulitas de corriente, con abundante contenido de materia orgánica particulada (fitodetrito), producidos por corrientes unidireccionales diluidas durante etapas activas de la descarga fluvial. El contenido icnológico comprende ejemplares de Haentzschelinia ottoi, Macaronichnus segregatis, Ophiomorpha irregulaire (?), Gyrochorte comosa y trazas de escape (fugichnia). El arreglo II muestra complejos de canales y barras integrados por areniscas medias a finas completamente reelaborados por ondulitas simétricas y estratificación entrecruzada de tipo hummocky, producto del retrabajo producido por flujos oscilatorios normales y de tormenta durante etapas de muy baja descarga fluvial. En estos arreglos, la icnofauna está dominada por Haentzschelinia ottoi y Skolithos linearis. El arreglo III corresponde a depósitos de sistemas de canales distributarios dominados por flujos hiperpícnicos, integrados por areniscas medias a finas que muestran un pasaje transicional y recurrente de estructuras sedimentarias tractivas, que incluyen gran concentración de materia orgánica particulada en sus caras de avalancha. Este último arreglo presenta trazas de escape o carece de bioturbación y se habría generado por corrientes de densidad fluvio-derivadas. Las dimensiones que muestran los sistemas de canales y barras que conforman el frente 
deltaico de la Formación Lajas son coincidentes con las determinadas en sistemas actuales y fósiles (Olariu y Bhattacharya, 2006; Buatois et al., 2012).

La integración de esta información permitió desarrollar un modelo esquemático en el que se explica la construcción y evolución del frente deltaico de la Formación Lajas (Fig. 9). En la etapa $T_{1}$ se produce la construcción y migración de las barras de desembocadura deltaica correspondiente al arreglo I, asociadas a múltiples canales distributarios terminales activos. Los niveles de fangolitas masivas con estructuras de deformación y laminadas, ricas en materia orgánica particulada son aportados desde una pluma hipopícnica por procesos de floculación y decantación (Fig. 9). En la etapa $T_{2}$ se produce la avulsión de las barras previamente formadas (arreglo I) y la construcción y migración de barras asociadas a las nuevas posiciones de los canales distributarios terminales activos (Fig. 9). En deltas fluvio-dominados la avulsión de las barras es un proceso frecuente debido a que los sedimentos aportados previamente por la descarga fluvial no son removidos por los procesos activos en la cuenca y, en consecuencia, las tasas de crecimiento de las barras son altas (Bhattacharya y Giosan, 2003; Olariu y Bhattacharya, 2006). Las barras abandonadas por los procesos de avulsión son reelaboradas por acción del oleaje (normal o de tormenta), dando lugar a las barras con arreglo II; la recurrencia de este tipo de barras evidencia la alta frecuencia de los procesos de avulsión. En la etapa $\mathrm{T}_{3}$, se produce el abandono de las barras generadas previamente y la construcción de barras de arreglo I asociadas a las nuevas posiciones que ocupan los canales distributarios terminales activos (Fig. 9). Este mecanismo de construcción que favorece la amalgamación y coalescencia de las barras arenosas es necesario para que se produzca la progradación del sistema deltaico. Las barras con arreglo III se producen durante descargas fluviales extraordinarias (flujos hiperpícnicos), y suelen presentarse truncando a las que presentan arreglos de tipo I y II.

\subsection{Parámetros de control sobre la fauna bentónica}

El análisis del contenido y distribución que muestran las trazas fósiles de la Formación Lajas en la localidad de Bajada de Los Molles permitió realizar inferencias paleoecológicas y paleoambientales de los diferentes subambientes que constituyen el sistema de delta estudiado (Tabla 2). Se reconocieron dos tipos de asociaciones icnológicas, la primera con una diversidad y abundancia moderada de trazas fósiles (offshore y shoreface), mientras que la segunda se caracteriza por una muy baja abundancia y diversidad de dichas estructuras (prodelta, frente deltaico y bahía interdistributaria). Asimismo, fueron identificados intervalos estratigráficos de espesor variable con ausencia de bioturbación (hiperpicnitas). La icnodiversidad y los índices de bioturbación determinados para cada una de las asociaciones de facies reconocidas (Tab. 2, Fig. 2), se diferencian de aquellos propuestos en trabajos previos (e.g., McIlroy et al., 2005; McIlroy, 2007). Se considera que estas diferencias se deben en primer lugar a las distintas interpretaciones paleoambientales, y más específicamente a las asociaciones de facies que se realizaron en este trabajo, que difieren de aquellas identificadas en los trabajos previos arriba mencionados. A continuación se analizan diversos factores paleoecológicos que habrían controlado el establecimiento y desarrollo de las comunidades bentónicas en estos ambientes.

\subsubsection{Salinidad}

Los estudios icnológicos constituyen una poderosa herramienta para reconstruir la paleosalinidad de un ambiente (Buatois et al., 1997). Los pasajes entre sistemas marinos normales y de transición se caracterizan por presentar diferentes gradientes de salinidad. A medida que la salinidad se aparta de las concentraciones marinas normales (35\%), se produce una marcada disminución en la diversidad de trazas fósiles y, en consecuencia, una mayor preservación de las estructuras sedimentarias físicas o mecánicas (MacEachern et al., 2005; Buatois y Mángano, 2011). Teniendo en cuenta trabajos previos en los cuales se describe el desarrollo de estructuras biogénicas en ambientes marinos normales y deltaicos (MacEachern et al., 2005; Knaust y Bromley, 2012; Coates y MacEachern, 2000; entre otros), los depósitos de la Formación Lajas muestran un empobrecimiento aún mayor en relación a lo esperable para estos ambientes. Dentro de este contexto, los depósitos de offshore y shoreface son los que muestran la existencia de condiciones de salinidad más próximas a las normales y en consecuencia la más alta diversidad y abundancia de trazas fósiles (Tabla 2). Sin embargo, la presencia de materia orgánica particulada en los depósitos de offshore indicaría cierta proximidad con un sistema fluvial activo. En el caso de los depósitos de prodelta, frente deltaico y bahía interdistributaria, la presencia de múltiples canales distributarios aportando plumas hipopícnicas (durante descargas fluviales normales), e hiperpícnicas (durante descargas 

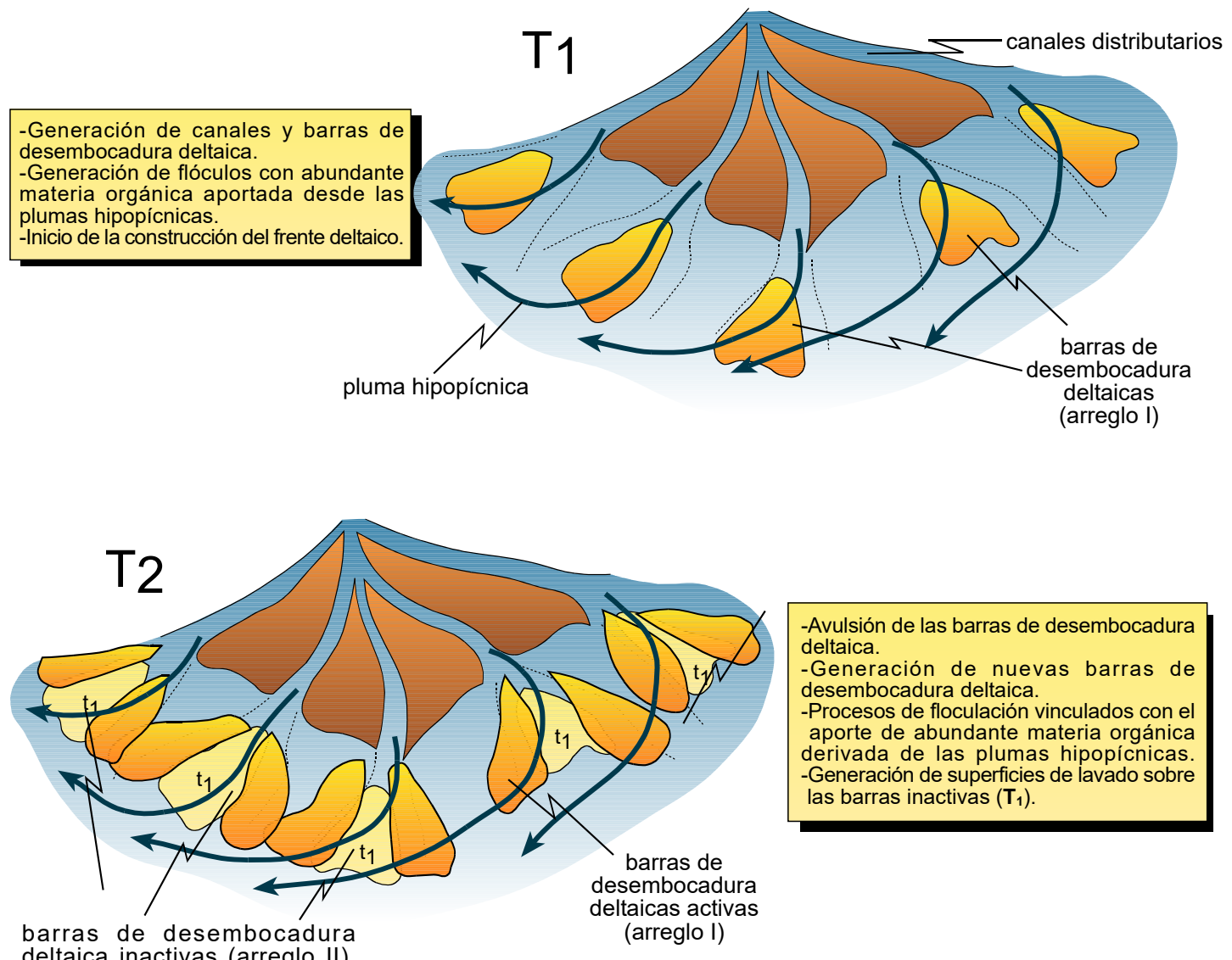

reelaboradas por acción de oleaje normal y de tormenta (superficies de lavado).

-Avulsión de barras de desembocadura deltaica.

-Generación de lóbulos de frente deltaico producto de la amalgamación de canales y barras de desembocadura deltaica.

-Procesos de floculación vinculados con el aporte de abundante materia orgánica desde las plumas hipopícnicas.

-Generación de superficies de lavado sobre

las barras inactivas $\left(\mathbf{T}_{1}-\mathbf{T}_{2}\right)$.

-Progradación del sistema.

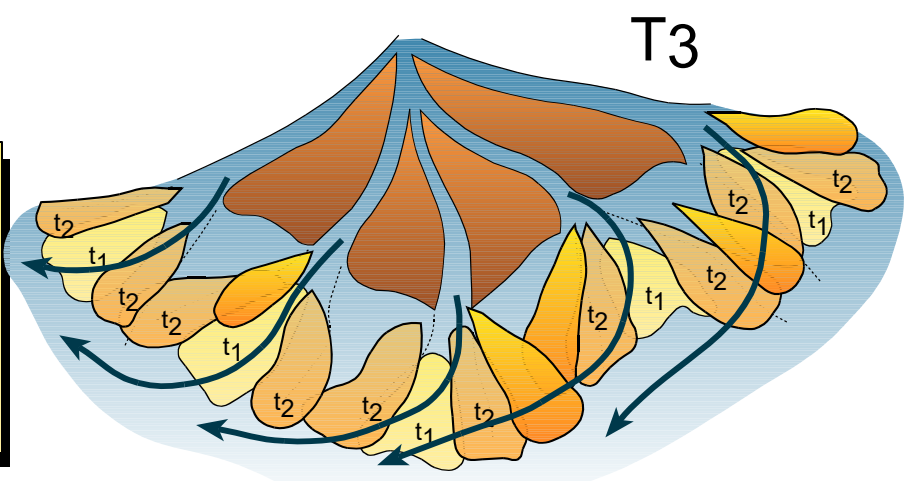

FIG. 9. Modelo esquemático propuesto para la construcción del frente deltaico de la Formación Lajas en el área de Bajada de Los Molles. $\mathbf{T}_{1}$ representa el inicio de la construcción del frente deltaico a partir del desarrollo de barras de desembocadura deltaica (arreglo I) asociadas a canales distributarios terminales. En $\mathbf{T}_{2}$ se produce la avulsión de las barras de desembocadura previamente construidas, continuando con la construcción del frente deltaico a partir de barras de desembocadura deltaica (arreglo I) que ocupan nuevas posiciones. En este momento las barras con arreglo I previamente construidas y abandonadas comienzan a ser retrabajadas por acción de oleaje (normal y de tormenta) dando lugar a barras con arreglo II. En $\mathbf{T}_{3}$ se continúan los procesos de construcción del frente deltaico con desarrollo de barras de desembocadura deltaica con arreglo I. Estos depósitos pueden ser erosionados por descargas de densidad hiperpícnicas dando lugar a barras con arreglo III. Modificado de Ponce et al. (2015). 
TABLA 2. PRINCIPALES CONTROLES PALEOECOLÓGICOS Y ABUNDANCIA QUE MUESTRAN LOS ICNOGÉNEROS EN LOS DIFERENTES SUBAMBIENTES ESTUDIADOS.

\begin{tabular}{|c|c|c|}
\hline Subambiente & Icnología & $\begin{array}{l}\text { Principales controles } \\
\text { paleoecológicos }\end{array}$ \\
\hline Offshore & $\begin{array}{c}\text { BI 5-6 en fangolitas y areniscas finas. Alta abundancia de Nereites isp., } \\
\text { Planolites isp., Teichichnus isp. y Thalassinoides suevicus. Baja abundancia } \\
\text { de Chondrites isp., Curvolithus simplex y Protovirgularia isp. } \\
\text { BI } 2 \text { en areniscas con óndulas simétricas. Baja abundancia de Skolithos } \\
\text { linearis. }\end{array}$ & energía hidrodinámica \\
\hline Shoreface & $\begin{array}{c}\text { BI 2-3 en areniscas. Alta abundancia de Haentzschelinia ottoi y } \\
\text { Macaronichnus segregatis. Baja abundancia de Arenicolites isp., } \\
\text { Gyrochorte comosa, Ophiomorpha irregulaire (?) y Skolithos linearis. }\end{array}$ & energía hidrodinámica \\
\hline Prodelta & $\begin{array}{c}\text { BI 1-2 en arreglos heterolíticos. Alta abundancia de Planolites isp. } \\
\text { Baja abundancia de Chondrites isp. y Thalassinoides suevicus. } \\
\text { BI 1-2 en areniscas con óndulas simétricas. Baja abundancia de Gyrochorte } \\
\text { comosa, Ophiomorpha irregulaire (?) y Skolithos linearis. }\end{array}$ & $\begin{array}{l}\text { salinidad } \\
\text { turbidez } \\
\text { oxigenación }\end{array}$ \\
\hline Frente deltaico & $\begin{array}{c}\text { BI } 2 \text { en areniscas. Alta abundancia de Haentzschelinia ottoi. } \\
\text { Baja abundancia de Asteriacites lumbricalis, Curvolithus simplex, } \\
\text { Gyrochorte comosa, Macaronichnus segregatis, Ophiomorpha irregulaire (?) } \\
\text { y trazas de escape. }\end{array}$ & $\begin{array}{l}\text { salinidad } \\
\text { tasa de sedimentación } \\
\text { energía hidrodinámica }\end{array}$ \\
\hline $\begin{array}{l}\text { Bahía } \\
\text { interdistributaria }\end{array}$ & $\begin{array}{l}\text { BI 2-3 en fangolitas. Alta abundancia rizolitos. Baja abundancia de } \\
\text { Chondrites isp., Thalassinoides suevicus, Phycosiphon incertum y } \\
\text { estructuras con laminación concéntrica (Asterosoma isp.). }\end{array}$ & salinidad turbidez \\
\hline Hiperpicnitas & $\begin{array}{c}\text { BI } 1 \text { en sistemas de canales distributarios dominados por flujos hiperpícnicos } \\
\text { de pequeña escala. Baja abundancia de trazas de escape. BI 1-2 en sistemas } \\
\text { de canal-albardón hiperpícnicos de gran escala. Baja abundancia de } \\
\text { Arenicolites isp., Skolithos linearis, Diplocraterion isp., estructuras con } \\
\text { laminación concéntrica y rizolitos. }\end{array}$ & $\begin{array}{l}\text { salinidad } \\
\text { turbidez } \\
\text { tasa de sedimentación } \\
\text { energía hidrodinámica }\end{array}$ \\
\hline
\end{tabular}

fluviales extraordinarias) habrían generado importantes variaciones de salinidad que, conjuntamente con la turbidez y la tasa de sedimentación, habrían afectado la distribución de los organismos bentónicos (Tabla 2). En el caso particular de la asociación de facies de bahía interdistributaria, las variaciones de salinidad se ven reflejadas en la frecuente presencia de grietas de sinéresis.

\subsubsection{Turbidez}

Como fuera descripto por MacEachern et al. (2005), un incremento de la turbidez puede reducir el índice de bioturbación y la diversidad de trazas fósiles en ambientes deltaicos. De esta manera, el aporte de sedimentos y materia orgánica particulada (fitodetrito) desde las plumas hipopícnicas e hiperpícnicas producen la acumulación, principalmente generada por procesos de floculación, de niveles de diferente espesor integrados por fango fluido. Estas variaciones de turbidez no sólo modifican las condiciones de vida de los organismos suspensívoros mediante la obstrucción de sus aparatos de filtración (Perkins, 1974; MacEachern et al., 2005), sino que también afectan los niveles de oxigenación del fondo, principalmente en la interfase agua-sedimento (Savrda y Bottjer, 1987; Gingras et al., 1998; entre otros). Los mayores efectos de la turbidez dentro de los subambientes reconocidos en la Formación Lajas ocurren en las asociaciones de facies de bahía interdistributaria, donde la presencia de niveles de fango fluido ricos en fitodetrito es dominante. En menor medida se reconocen niveles de fango fluido ricos en fitodetrito en las asociaciones de facies de prodelta. En ambos casos, los niveles de fango fluido se relacionan con el desarrollo de plumas hiperpícnicas e hipopícnicas. Ambas plumas aportan un gran volumen de sedimento fino y materia orgánica particulada en suspensión (fitodetrito), situación que se ve reflejada en la presencia recurrente de paredes construccionales completamente tapizadas por pelitas y restos vegetales.

\subsubsection{Tasa de sedimentación}

Los organismos vivos presentan distintas capacidades de adaptación a las rápidas variaciones en la tasa de sedimentación que caracteriza a los 
sistemas de transición (Olariu y Bhattacharya, 2006; Tonkin, 2012). En este sentido, son diversos los trabajos que muestran la capacidad de algunos organismos a sobrevivir en equilibrio con estas variaciones (Pemberton et al., 2001; Howell et al., 2007). El concepto de ventana de colonización, propuesto por Pollard et al. (1993), que refiere al tiempo disponible para la ocupación del sustrato entre eventos de depositación, permite entender cómo funciona la colonización del sustrato en ambientes de diferente energía. En las facies de offshore analizadas, asociadas a una constante pero baja tasa de sedimentación, la obliteración de las estructuras sedimentarias en el sustrato por parte de los organismos es importante, ya que la ventana de colonización se mantiene continua a través del tiempo. Contrariamente, en las asociaciones de facies de frente deltaico, la presencia de una gran cantidad de canales distributarios terminales produce variaciones significativas en la tasa de sedimentación, las cuales están vinculadas con las etapas de descarga normal y de crecida (corrientes de densidad hiperpícnicas) de los sistemas fluviales. En ambas situaciones la tasa de sedimentación es moderada a alta y la bioturbación se restringe sólo al tope de los paquetes sedimentarios, donde los procesos de oleaje normal y de tormenta reelaboran las barras, desarrollando ventanas de colonización de corta duración para el establecimiento de la endofauna.

\subsubsection{Energía hidrodinámica}

Las variaciones energéticas reconocidas en los diferentes subambientes influyen significativamente sobre el desarrollo de los organismos bentónicos (Buatois y Mángano, 2011). Las asociaciones de facies de prodelta y bahía interdistributaria se encuentran dominadas por trazas asignadas a organismos detritívoros, mientras que en las asociaciones de facies de shoreface el establecimiento de organismos suspensívoros se ve favorecido por las condiciones de energía moderada a alta y buena oxigenación del sustrato, debido a la acción de oleaje normal y de tormenta. En los depósitos del frente deltaico, las barras que presentan estratificación entrecruzada tangencial y en artesa de gran escala, estratificación de tipo hummocky y ondulitas simétricas, muestran trazas asignadas a Haentzschelinia ottoi, Macaronichnus segregatis y Ophiomorpha irregulaire (?), todas ellas interpretadas como el producto de la actividad de organismos tolerantes a condiciones de energía moderada a alta. Las condiciones de mayor energía y alta tasa de sedimentación en el sistema ocurren en las asociaciones de facies hiperpícnicas, las cuales muestran de manera frecuente intervalos estratigráficos sin bioturbación y, en menor medida, con trazas de escape.

\section{Modelo depositacional}

La integración de los datos sedimentológicos e icnológicos permitió reconocer la presencia de asociaciones de facies sedimentarias e icnofacies propias de un sistema deltaico fluvio-dominado reelaborado por descargas hiperpícnicas y acción de oleaje (normal y de tormenta). Se reconoció que los depósitos estuvieron controlados tanto por procesos alogénicos como autigénicos. Los procesos alogénicos controlaron el arreglo que muestra la sucesión analizada, el cual es progradante en su tramo basal, donde el aporte de sedimentos habría superado a la generación de espacio de acomodación, y agradante en su tramo medio y superior, donde existió un equilibrio entre el aporte de sedimentos al sistema y la generación de espacio de acomodación. El arreglo general de la sucesión analizada habría estado principalmente controlado por el basculamiento de hemigrábenes heredados del Precuyano, y reactivados por la actividad tectónica en la zona de la Dorsal de Huincul (Freije et al., 2002). Los procesos autigénicos controlaron el mecanismo de construcción del frente deltaico, integrado por pequeños canales distributarios terminales, los cuales son rápidamente colmatados por agradación o migración lateral de las barras de desembocadura, produciendo la avulsión y el desarrollo de un complejo de canales y barras de desembocadura a ambos lados de la barra previamente acumulada. La recurrencia de este mecanismo de acumulación produce un frente deltaico integrado por complejos de canales y barra amalgamados, con importantes variaciones temporales y espaciales de facies sedimentarias, como fue también documentado por Olariu y Bhattacharya (2006) en diferentes sistemas deltaicos actuales y fósiles.

Desde una perspectiva paleoambiental, la Formación Lajas en la localidad de Bajada de Los Molles se inicia con depósitos de offshore que pasan, en transición, a depósitos de shoreface que conforman un arreglo netamente progradante y de poco espesor (Fig. 2 y $_{1}$ en Fig. 10). Las asociaciones de facies 


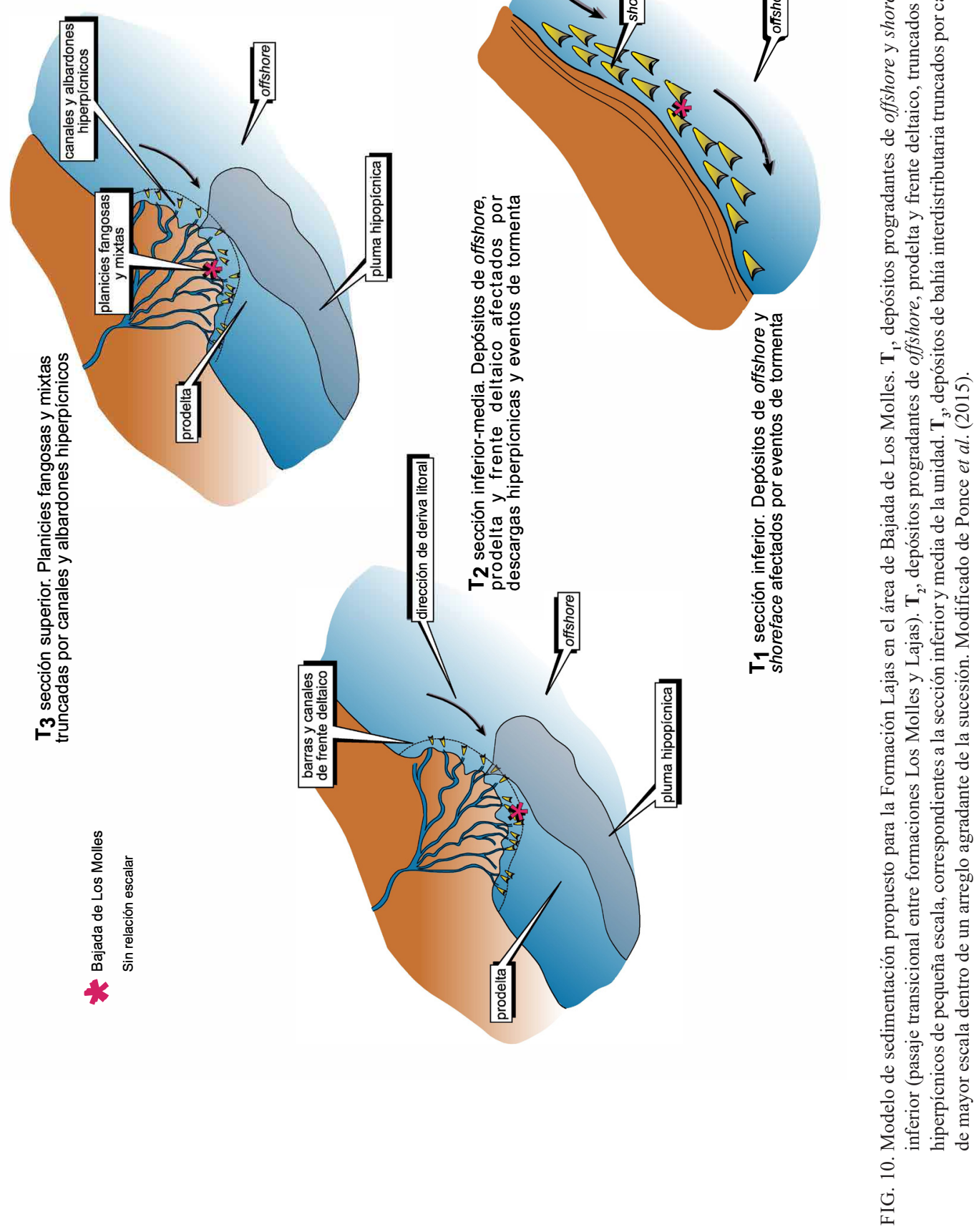


de offshore-shoreface pasan en transición hacia depósitos de prodelta y hacia canales y barras de frente deltaico. En estos depósitos se reconocen icnofacies de Cruziana y Skolithos empobrecidas (Fig. 2 $\mathrm{y}_{2}$ en Fig. 10). Los depósitos de prodelta y frente deltaico presentan abundante cantidad de materia orgánica particulada (fitodetrito), muy baja diversidad y abundancia de trazas fósiles, y un gran número de complejos de canales y barras amalgamados, con gran dispersión en sus paleocorrientes. Todas estas características sugieren un origen fluvio-dominado para estos depósitos (Olariu y Bhattacharya, 2006; Canale et al., 2015). De manera frecuente estos depósitos se encuentran truncados por sistemas de canales distributarios dominados por flujos hiperpícnicos de pequeña escala. Finalmente, hacia el techo de la sucesión analizada se reconoce la presencia de depósitos de bahía interdistributaria (Fig. 2 y $_{3}$ en Fig. 10). Estos depósitos se encuentran erosionados por sistemas de canales y albardones dominados por flujos hiperpícnicos de gran escala, que aportan un gran volumen de sedimentos finos y materia orgánica a las bahías interdistributarias.

\section{Conclusiones}

1. Las evidencias sedimentológicas e icnológicas aportadas en esta contribución permitieron reinterpretar el origen de los depósitos de la Formación Lajas en la localidad de Bajada de Los Molles. Estos depósitos, que habían sido previamente asignados a deltas dominados por marea o a deltas fluvio-dominados con acción de marea, son aquí interpretados como correspondientes a un sistema deltaico fluvio-dominado afectado por descargas de densidad hiperpícnicas.

2. El principal mecanismo de construcción del frente deltaico ocurre por el apilamiento y la coalescencia de complejos de canales distributarios terminales y barras de desembocadura deltaica. Estos complejos de canales distributarios terminales y barras experimentan avulsiones rápidas provocadas por procesos autigénicos.

3. Los depósitos de offshore-shoreface se reconocieron a la base de la sucesión estratigráfica y son los que muestran la mayor diversidad y abundancia de trazas fósiles. Los depósitos de prodelta, frente deltaico y bahía interdistributaria han sido reconocidos en la sección media y superior y muestran una moderada diversidad y abundancia de trazas fósiles, mientras que las hiperpicnitas muestran ausencia de trazas fósiles o pequeños intervalos estratigráficos con presencia de trazas de escape.

4. Las hiperpicnitas muestran dos arreglos distintivos. Uno corresponde a sistemas de canales distributarios dominados por flujos hiperpícnicos de pequeña escala, que internamente se integran de areniscas que muestran un pasaje transicional y recurrencia de estructuras sedimentarias y una gran concentración de materia orgánica particulada (fitodetrito), principalmente en las caras de avalancha de la estructuras tractivas. Este arreglo se encuentra erosionando a los sistemas de prodelta y frente deltaico. El otro arreglo corresponde a sistemas de canales y albardones dominados por flujos hiperpícnicos de gran escala, que internamente se encuentran integrados por conglomerados y areniscas con pasajes transicionales y recurrentes de estructuras sedimentarias tractivas o masivas, con presencia de troncos, y en menor medida materia orgánica particulada (fitodetrito). Estos arreglos han sido reconocidos truncando los depósitos de bahía interdistributaria.

5. Los principales factores de control en el contenido de trazas fósiles habrían sido las fluctuaciones en la salinidad, turbidez, tasa de sedimentación y energía hidrodinámica.

\section{Agradecimientos}

Este estudio fue financiado por CONICET PIP 417 y Proyecto de Vinculación Tecnológica RSPU 3270/13 y PUE 0031CO a JJP, y PI UNRN 40-A-616 a NBC. Los autores desean agradecer el apoyo brindado por el Instituto de Investigación en Paleobiología y Geología (IIPG) de la Universidad Nacional de Río Negro, al Dr. A. Wetzel por las discusiones en campo y por las sugerencias bibliográficas, y a D. Campetella por la ayuda con la edición de las figuras. También agradecemos a los revisores Dres. L.A. Buatois y R.M. Vega, al editor W. Vivallo Sandoval y sus asistentes, cuyos comentarios y recomendaciones aportaron sustancialmente al enriquecimiento de esta contribución.

\section{Referencias}

Arnott, R.W.; Southard, J.B. 1990. Exploratory flow-duct experiments on combined-flow bed configurations, and some implications for interpreting storm event stratification. Journal of Sedimentary Petrology 60 : 211-219. 
Bann, K.L.; Fielding, C.R. 2004. An integrated ichnological and sedimentological comparison of non-deltaic shoreface and subaqueous delta deposits in Permian reservoir units of Australia. In The Application of Ichnology to Palaeoenvironmental and Stratigraphic Analysis (McIlroy, D.; editor). The Geological Society of London, Special Publication 228: 273-310. doi: 10.1144/GSL.SP.2004.228.01.13.

Berdini, O.; Arregui, C.; Pimentel, M.M. 2004. Evolución tecto-sedimentaria de la estructura Río Neuquén, cuenca Neuquina, República Argentina. In Congreso Geológico Argentino, No. 15, Actas en CD. El Calafate.

Bhattacharya, J.P. 2006. Deltas. In Facies Models revisited (Posamentier, H.W.; Walker, R.G.; editors). Society of Economic Paleontologists and Mineralogists Special Publication 84: 237-292.

Bhattacharya, J.P.; Giosan, L. 2003. Wave-influenced deltas, geomorphological implications for facies reconstruction. Sedimentology 50: 187-210.

Bhattacharya, J.P.; MacEachern, J.A. 2009. Hyperpycnal rivers and prodeltaic shelves in the Cretaceous seaway of North America. Journal of Sedimentary Research 79: 184-209.

Boyd, R.; Dalrymple, R.; Zaitlin, B.A. 1992. Clasification of clastic coastal depositional environments. Sedimentary Geology 80: 139-150.

Bracaccini, C.I. 1970. Rasgos tectónicos de las acumulaciones mesozoicas en las provincias de Mendoza y Neuquén. Revista de la Asociación Geológica Argentina 25: 275-284.

Buatois, L.; Mángano, M.G. 2011. Ichnology: organismsubstrate interactions in space and time. Cambridge University Press: 358 p. Cambridge.

Buatois, L.A.; Saccavino, L.L.; Zavala, C. 2011. Ichnologic signatures of hyperpycnal flow deposits in Cretaceous river-dominated deltas, Austral Basin, southern Argentina. In Sediment transfer from shelf to deep water-Revisiting the delivery system (Slatt R.M.; Zavala C.; editors). American Association of Petroleum Geologists Studies in Geology 61: 153-170.

Buatois, L.A.; Mángano, M.G.; Maples, C.G.; Lanier, W.P. 1997. The paradox of nonmarine ichnofaunas in tidal rhythmites: Integrating sedimentologic and ichnologic data from the Late Carboniferous of Eastern Kansas. Palaios 12: 467-481. EE.UU.

Buatois, L.A.; Santiago, N.; Herrera, M.; Plink-Bjôrklund, P.; Steel, R.; Espin, M.; Parra, K. 2012. Sedimentological and ichnological signatures of changes in wave, river and tidal influence along a Neogene tropical deltaic shoreline. Sedimentology 59: 1568-1612.
Canale, N.; Ponce, J.J.; Bournod, C.N.; Martínez, M.A.; Carmona, N.B. 2013. Ondas de sedimento de grano fino generadas por corrientes hiperpícnicas en deltas fluviodominados de la Formación Lajas (Jurásico Medio), Cuenca Neuquina, Argentina. In Simposio Argentino Jurásico, No. 5. Ameghiniana 50 (4) SuplementoResúmenes: 15 p. Argentina.

Canale, N.; Ponce, J.J.; Carmona, N.; Drittanti, D.; Olivera, D.; Martínez, M.A.; Bournod, C.N. 2015. Sedimentología e Icnología de deltas fluvio-dominados afectados por descargas hiperpícnicas de la Formación Lajas (Jurásico Medio), Cuenca Neuquina, Argentina. Andean Geology 42: 114-138.

Canale, N.; Ponce, J.J.; Carmona, N.B.; Drittanti, D.I. 2016. Ichnology of deltaic mouth-bar systems of the Lajas Formation (Middle Jurassic) in the Sierra de la Vaca Muerta, Neuquén Basin, Argentina. Ameghiniana 53: $170-183$.

Carbone, C. 1988. Sismoestratigrafía del grupo Cuyo Inferior en la Cuenca Neuquina. Boletín de Informaciones Petroleras: 67-87.

Carmona, N.B.; Ponce, J.J. 2011. Ichnology and sedimentology of Miocene hyperpycnites of the Austral foreland basin (Tierra del Fuego, Argentina): Trace fossil distribution and paleoecological implications. In Sediment transfer from shelf to deep water-Revisiting the delivery system (Slatt R.M.; Zavala, C.; editors). American Association of Petroleum Geologists Studies in Geology 61: 171-192.

Clifton, H.E.; Thompson, J.K. 1978. Macaronichnus segregatis: A feeding structure of shallow marine polychaetes. Journal of Sedimentary Petrology 48: 1293-1302.

Coates, L.; MacEachern, J.A. 1999. The ichnological signature of wave-and river-dominated deltas: Dunvegan and Basal Belly River formations, West-Central Alberta. In Digging Deeper, Finding a Better Bottom Line (Wrathall, B.; Johnston, G.; Arts, A.; Rozs, L.; Zonneveld, J.P.; Arcuri, D.; McLellan, S.; editors). Canadian Society of Petroleum Geologists and Petroleum Society, Core Conference, Paper 99: p. 114C.

Coates, L.; MacEachern, J.A. 2000. Integrating ichnology and sedimentology to differentiate between riverdominated deltas, wave-dominated deltas, and shorefaces, examples from the Cretaceous of western Canada. Geological Society of America, Cordilleran Section, 96th Annual Meeting, Vancouver, British Columbia 32: p. A7.

Dasgupta, S.; Buatois, LA. 2012. Unusual occurrence and stratigraphic significance of the Glossifungites 
ichnofacies in a submarine paleo-canyon-Example from a Pliocene shelf-edge delta, Southeast Trinidad. Sedimentary Geology 269-270: 69-77.

Dasgupta, S.; Buatois, L A.; Mángano M.G. 2016. Living on the edge: evaluating the impact of stress factors on animal-sediment interactions in subenvironments of a shelf-margin delta, the Mayaro Formation, Trinidad. Journal of Sedimentary Research 86: 1034-1066.

De Ferraríis, C. 1947. Edad del arco o dorsal antiguo del Neuquén oriental, de acuerdo con la estratigrafía de la zona inmediata. Asociación Geológica Argentina. Buenos Aires. Revista 2: 256-283.

Dean, J.S. 1987. Depositional environments y paleogeography of the lower to Middle Jurassic Cuyan Group, Neuquén Basin, Argentina. Ph.D. Thesis (Unpublished), Department of Geology and Geological Engineering, Colorado School of Mines: 587 p. Golden, Colorado.

Dellapé, D.A.; Mombrú, C.; Pando, G.A.; Riccardi, A.C.; Uliana, M.A.; Westermann, G.E.G. 1979. Edad y correlación de la Formación Tábanos en Chacay Melehue y otras localidades de Neuquén y Mendoza. Obra del Centenario del Museo de La Plata 5: 1-106.

Digregorio, J.H. 1978. Estratigrafía de las acumulaciones mesozoicas. In Geología y Recursos Naturales del Neuquén (Rollen, E.; editor). Congreso Geológico Argentino, No. 7, Relatorio: 37-65. Buenos Aires.

Digregorio, R.E.; Gulisano, C.A.; Gutierrez Pleimling, A.R.; Minitti, S.A. 1984. Esquema de la evolución geodinámica de la cuenca Neuquina y sus implicaciones paleogeográficas. In Congreso Geológico Argentino, No. 9, Actas 2: 147-162. San Carlos de Bariloche.

Franzese, J.R.; Spalletti, L.A. 2001. Late Triassic-early Jurassic continental extension in southwestern Gondwana: tectonic segmentation and pre-break-up rifting. Journal of South American Earth Sciences 14: 257-270.

Freije, H.; Azúa, G.; González, R.; Ponce, J.J.; Zavala, C. 2002. Actividad tectónica sinsedimentaria en el Jurásico del sur de la Cuenca Neuquina. In Congreso de Exploración y Desarrollo de Hidrocarburos, No. 5, Actas: 17. Mar del Plata.

Galloway, W.E. 1975. Process framework for describing the morphologic and stratigraphic evolution of deltaic depositional systems. In Deltas, Models for Exploration (Broussard, M.L.; editor). Houston Geological Society: 87-89. Houston.

Gani, M.R.; Bhattacharya, J.P.; MacEachern, J.A. 2004. Using ichnology to determine relative influence of waves, storms, tides and rivers in deltaic deposits: examples from Cretaceous delta complexes in the Western Interior Seaway, Wyoming-Utah, USA. In American Association of Petroleum Geologists, Annual meeting, Abstracts 13: p. A49. Dallas.

Gingras, M.K.; MacEachern, J.A.; Pemberton, S.G. 1998. A comparative analysis of the ichnology of wave-and river-dominated allomembers of the Upper Cretaceous Dunvegan Formation. Bulletin of Canadian Petroleum Geology 46: 51-73.

Gugliotta, M.; Flint, S.S.; Hodgson, D.M.; Veiga, G.D. 2015. Stratigraphic record of river-dominated crevasse subdeltas with tidal influence (Lajas Formation, Argentina). Journal of Sedimentary Research 85: 265-284.

Gulisano, C.A.; Pando, G.A. 1981. Estratigrafía y facies de los depósitos jurásicos entre Piedra del Aguila y Sañico, Departamento Collon Cura, Provincia del Neuquén. In Congreso Geológico Argentino, No 8, Actas 3: 553-577. San Luis.

Gulisano, C.A.; Gutiérrez Pleimling, A.R. 1995. The Jurassic of the Neuquén Basin: Mendoza Province. Guía de Campo. Asociación Geológica Argentina, Publicación Especial 159: 103 p.

Gulisano, C.A.; Gutiérrez Pleimling, A.R.; Digregorio, R.E. 1984. Esquema estratigráfico de la secuencia jurásica al este de la provincia del Neuquén. In Congreso Geológico Argentino, No. 9, Actas 1: 236-259.

Harms, J.C.; Southard, J.B.; Spearing, D.R.; Walker, R.G. 1975. Depositional environments as interpreted from primary sedimentary structures and stratification sequences. Society for Sedimentary Geology (SEPM), Short Course Notes 2: $161 \mathrm{p}$.

Howell, J.; Schwarz, E.; Spalletti, L.; Veiga, G. 2005. The Neuquén Basin: An overview. In The Neuquén Basin: a Case Study in Sequence Stratigraphy and Basin Dynamics (Veiga, G.; Spalletti, L.; Howell, J.; Schwarz, E.; editors). Geological Society of London, Special Publication 252: 1-14. Londres.

Howell, C.D. Jr.; Bhattacharya, J.P.; MacEachern, J.A. 2007. Bedset sedimentation rates in a Turonian Greenhouse delta front, Wall Creek Member, Frontier Formation, Powder Reiver Basin, Wyoming. In Ichnological Application to Sedimentological and Sequence Stratigraphic Problems (MacEachern, J.A.; Gingras, M.K.; Bann, K.L.; Pemberton, S.G.; editors). Society for Sedimentary Geology Research Conference, Price: 81-84.

Jeon, C.; Park, J.H.; Varlamov, S.M.; Yoon, J.-H.; Kim, Y.H.; Seo, S.; Park, Y.G.; Min, H. S.; Lee, J. H.; Kim, C.H. 2014. Seasonal variation of semidiurnal internal 
tides in the East/Japan Sea, Journal of Geophysical Research: Oceans: 2843-2859.

Knaust, D.; Bromley, R. 2012. Trace Fossils as Indicators of Sedimentary Environments. Developments in Sedimentology 64. Elsevier: 924 p.

Leanza, H.A.; Hugo, C.A.; Repol, D. 2001. Hoja Geológica 3969-I, Zapala, Provincia del Neuquén. Instituto de Geología Recursos Minerales, Servicio Geológico Minero Argentino. Boletín 275: 128 p. Buenos Aires.

Legarreta, L.; Gulisano, C.A. 1989. Análisis estratigráfico secuencial de la Cuenca Neuquina (Triásico superiorTerciario inferior). In Cuencas Sedimentarias Argentinas. Serie Correlación Geológica (Chebli, G.; Spaletti, L.A.; editors). Universidad Nacional de Tucumán: 221-243. San Miguel de Tucumán.

Lokho, K.; Singh, B.P.; Whiso, K.; Ezung, O.C. 2017. Ichnology of the Laisong Formation (late Eoceneearly Oligocene) of the Naga Hills, Indo-Burma range (IBR): Paleoenvironmental significance. Journal of Asian Earth Sciences 162: 13-24.

MacEachern, J.A.; Bann, K.L.; Bhattacharya, J.P.; Howell, C.D. 2005. Ichnology of deltas: organism responses to the dynamic interplay of rivers, waves, storms and tides. In River Deltas: Concepts, Models and Examples (Bhattacharya, J.P.; Giosan, L.; editors). Society of Economic Paleontologists and Mineralogists. Special Publication 83: 49-85.

McIlroy, D. 2007. Palaeoenvironmental controls on the ichnology of tide-influenced facies with an example from a macrotidal tide-dominated deltaic depositional system, Lajas Formation, Neuquén Province, Argentina. In Sediment-Organism Interactions: a Multifaceted Ichnology (Bromley, R.; Buatois, L.; Mángano, G.; Genise, J.; Melchor, R.; editors). Society for Sedimentary Geology (SEPM), Special Publication 88: 195-212.

McIlroy, D.; Flint, S.; Howell, J.A.; Timms, N. 2005. Sedimentology of the Jurassic tide-dominated Lajas Formation, Neuquén Basin, Argentina. In The Neuquén Basin, Argentina: a Case Study in Sequence Stratigraphy and Basin Dynamics (Veiga, G.; Spalletti, L.; Howell, J.; Schwarz, E.; editors). Geological Society of London, Special Publication 252: 83-108. London.

Mikhailov, V.N.; Mikhailova, M.V. 2010. Delta formation processes at the Mississippi River mouth. Water Resources 37: 595-610.

Mosquera, A.; Ramos, V.A. 2006. Intraplate deformation in the Neuquén Embayment. In Evolution of an Andean margin: a tectonic and magmatic view from the Andes to the Neuquén Basin (35-39 S lat.) (Kay, S.; Ramos, V.; editors). Geological Society of America, Special Paper 407: 97-123.

Mulder, T.; Syvitski, J.P.M.; Migeon, S.; Faugères, J.C.; Savoye, B. 2003. Marine hyperpycnal flows: initiation, behavior and related deposits: A review. Marine and Petroleum Geology 20: 861-882.

Olariu, C.; Bhattacharya, J.P. 2006. Terminal distributary channels and delta front architecture of river-dominated delta systems. Journal of Sedimentary Research 76: 212-233.

Olivero, E.B.; López, M.I.; Malumián, N.; Torres Carbonell, P. J. 2010. Eocene graphoglyptids from shallow-marine, high energy, organic-rich, and bioturbated turbidites, Fuegian Andes, Argentina. Acta Geológica Polonica 60 (1): 77-91.

Orchuela, I.; Ploszkiewicz, J.V.; Viñes, R. 1981. Reinterpretación estructural de la denominada "Dorsal Neuquina”. In Congreso Geológico Argentino, No. 8, Actas 3: 281-293. San Luis.

Paim, P.S.G.; Silveira, A.; Lavina, E.; Faccini, U.; Leanza, H.; Teixeira de Oliveira, J.M.M.; D’avila, R. 2008. Highresolution stratigraphy and gravity flow deposits in the Los Molles Formation (Cuyo Group-Jurassic) at La Jardinera Region, Neuquén Basin. Revista de la Asociación Geológica Argentina 63: 728-753.

Pángaro, F.; Pereira, D.M.; Miccuci, E. 2009. El Sinrift de la Dorsal de Huincul, Cuenca Neuquina: Evolución y control sobre la estratigrafía y estructura del área. Revista de la Asociación Geológica Argentina 65: 265-277.

Pemberton, S.G.; Spila, M.; Pulham, A.J.; Saunders,T.; MacEachern, J.A.; Robbins, D.; Sinclair, I.K. 2001. Ichnology and sedimentology of shallow to marginal marine systems: Ben Nevis and Avalon Reservoirs, Jeanne d'Arc Basin. Geological Association of Canada, Short Course Notes 15: 343 p.

Perkins, E.J. 1974. The Biology of Estuaries and Coastal Waters. Academic Press: 678 p. London.

Plink-Björklund, P.; Steel, R. 2004. Initiation of turbidity currents: Outcrop evidence for Eocene hyperpycnal flow turbidites. Sedimentary Geology 165 (1-2): 9-52.

Ploszkiewicz, J.V.; Orchuela, I.A.; Vaillard J.C.; Viñes, R.F. 1984. Compresión y desplazamiento lateral en la zona de falla Huincul, estructuras asociadas, Provincia del Neuquén. In Congreso Geológico Argentino, No. 9, Actas 2: 163-169. San Carlos de Bariloche.

Plummer, P.S.; Gostin, V.A. 1981. Shrinkage cracks: desiccation or synaeresis. Journal of Sedimentary Petrology 54: 1147-1156.

Pollard, J.E.; Goldring, R.; Buck, S.G. 1993. Ichnofabrics containing Ophiomorpha: significance in shallow- 
water facies interpretation. Journal of the Geological Society of London 150: 149-164.

Ponce, J.J.; Carmona, N.B. 2011a. Miocene deep-marine hyperpycnal channel levee complexes, Tierra del Fuego, Argentina: Facies associations and architectural elements. In Sediment transfer from shelf to deep water. Revisiting the delivery system (Slatt, R.M.; Zavala, C.; editors). American Association of Petroleum Geologists. Studies in Geology 61: 75-93.

Ponce, J.J.; Carmona, N.B. 2011 b. Coarse-grained sediment waves in hiperpycnal clinoform systems, Miocene of the Austral foreland basin, Argentina. Geology 39: 763-766.

Ponce, J.J.; Olivero, E.B.; Martinioni, D.R.; López Cabrera, M.I. 2007. Sustained and episodic gravity flow deposits and related bioturbation patterns in Paleogene turbidites (Tierra del Fuego, Argentina). In Sediment-organism interactions: a multifaceted ichnology (Bromley, R.G.; Buatois, L.A.; Mángano, M.G.; Genise, J.F.; Melchor, R.N.; editors). Society for Sedimentary Geology (SEPM). Special Publication 88: 253-266.

Ponce, J.J.; Canale, N.; Carmona, N.B.; Bournod, C.N.; Martínez, M.A.; Olivera, D.E. 2012. Sedimentología e icnología en sucesiones hiperpícnicas de la Formación Lajas, área de Portada Covunco, Neuquén, Argentina. In Reunión Argentina de Sedimentología, No. 13, Asociación Argentina de Sedimentología, Resúmenes: 175-176. Salta.

Ponce, J.J.; Montagna, A.O.; Carmona, N.B.; Canale, N. (Editores). 2015. Guía de Campo. Escuela de Verano 2015. Sedimentología e Icnología de los Sistemas Petroleros no Convencionales de la Cuenca Neuquina (Los Molles-Lajas y Vaca Muerta-Quintuco). Universidad Nacional de Río Negro-Fundación YPF: $112 \mathrm{p}$.

Rosenfeld, U.; Volkheimer W. 1979. Types of paleoenvironments of the Middle Jurassic Lajas Formation (Neuquén, Argentina). In International Gondwana Symposium, 1977. Hindustan Publishing Corporation: 238-247. Delhi.

Rossi, V.M.; Steel, R.J. 2016. The role of tidal, wave and river currents in the evolution of mixed-energy deltas: Example from the Lajas Formation (Argentina). Sedimentology 63: 824-864.

Savrda, C.E.; Bottjer, D.J. 1987. The exaerobic zone, a new oxygen deficient marine biofacies. Nature 327 : 54-56.

Shaw, J.B.; Mohrig, D. 2014. The importance of erosion in distributary channel network growth, Wax Lake
Delta, Louisiana, USA. Geology 42 (1): 31-34. doi: 10.1130/G34751.1.

Spalletti, L.A.; Franzese, J.R.; Veiga, G.D.; Schwarz, E. 2005. The Mesozoic evolution of the Neuquén Basin and its relationship with the history of Western Gondwana. In Gondwana Conference, No. 12, Abstracts: 343 p. Mendoza.

Swift, D.J.P.; Gregory, H.; Vincent, C.E. 1986. Fluid processes and sea-floor response on a modern stormdominated shelf: middle Atlantic Shelf of North America. Part 1: The storm-current regime. In Shelf Sands and Sandstones (Knight, R.J.; McLean, J.R.; editors). Canadian Society of Petroleum Geologists 2 : 99-119.

Taylor, A.M.; Goldring, R. 1993. Description and analysis of bioturbation and ichnofabric. Journal of the Geological Society, London 150: 141-148.

The Open University. 1999. Waves, Tides and Shallow Water Processes, second edition. Butterworth-Heinemann: 227 p. Oxford.

Tonkin, N.S. 2012. Deltas. In Trace fossils as indicators of sedimentary environments (Bromley, R.G.; Knaust, D.; editors). Developments in Sedimentology 64: 507-528.

Uliana, M.A.; Legarreta, L. 1993. Hydrocarbon habitat in a Triassic-to-Cretaceous Sub-Andean setting Neuquén Basin, Argentina, Journal of Petroleum Geology 16: 397-420.

Uliana, M.A.; Biddle, K.T.; Cerdan, J. 1989. Mesozoic extension and the formation of Argentine sedimentary basin. In Extensional Tectonics y Stratigraphy of the North Atlantic Margins (Tankard, A.J.; Balkwill, H.R.; editors). American Association of Petroleum Geology (AAPG). Memoirs 46: 509-614.

Veiga, G.D. 2000. Estratigrafía y Sedimentología de la Formación Challacó, Cuenca Neuquina Austral, República Argentina. Tesis Doctoral (Inédito), Universidad Nacional de La Plata, Facultad de Ciencias Naturales y Museo: 265 p.

Vergani, G.D. 2005. Control estructural de la sedimentación jurásica (Grupo Cuyo) en la Dorsal de Huincul, Cuenca Neuquina. Modelo de falla lístrica rampa-plano invertida. Boletín de Informaciones Petroleras 1 : 32-42. Oxford.

Vergani, G.D.; Tankard, A.J.; Belotti, H.J.; Welsink, H.J. 1995. Tectonic evolution and paleogeography of the Neuquén Basin, Argentina. In Petroleum Basins of South America (Tankard, A.J.; Suárez, R.; Welsink, H.; editors). American Association of Petroleum Geologists (AAPG). Memoirs 62: 383-402. 
Vicente, J.C. 2005. Dynamic paleogeography of the Jurassic Andean Basin: pattern of transgression and localization of main straits through the magmatic arc. Revista de la Asociación Geológica Argentina 60: 221-250.

Vieira de Luca, P.H.; Basilici, G. 2013. A prodeltaic system controlled by hyperpycnal flows and storm waves: reinterpretation of the Punta Negra Formation (Lower-Middle Devonian, Argentine Precordillera). Brazilian Journal of Geology 43: 673-694.

Weaver, C.E. 1931. Paleontology of the Jurassic and Cretaceous of West Central Argentina. University of Washington Memoir I: 496 p. Washington.

Yrigoyen, M.R. 1991. Hydrocarbon resources of Argentina. In World Petroleum Congress, No. 13, Petrotecnia, Special Issue: 38-54. Buenos Aires.

Zavala, C.A. 1993. Estratigrafía y análisis de facies de la Formación Lajas (Jurásico medio) en el sector suroccidental de la Cuenca Neuquina. Provincia del Neuquén. República Argentina. Tesis Doctoral (Inédito), Universidad Nacional del Sur, Departamento de Geología: 249 p.

Zavala, C.A.; González, R. 2001. Estratigrafía del Grupo Cuyo (Jurásico inferior-medio) en la Sierra de la Vaca Muerta, Cuenca Neuquina. Boletín de Informaciones Petroleras 65: 52-64.

Zavala, C.; Ponce, J.; Marteau, M. 2001. Origin, sequence stratigraphy and hydrocarbon potential of the Rayoso Formation (Aptian-Albian) in the central Neuquén Basin (Argentina). American Association of Petroleum Geologists (AAPG) Hedberg Conference "New Technologies and New Play Concepts in Latin America", Abstracts: 35-36. Mendoza.

Zavala, C.; Ponce, J.J.; Arcuri, M.; Drittanti, D.; Freije, H.; Asensio, M. 2006. Ancient Lacustrine hyperpycnites: a depositional model from a case study in the Rayoso Formation (Cretaceous) of west-central Argentina. Journal of Sedimentary Research 76: 40-58.

Manuscrito recibido: Abril 22, 2018; revisado/aceptado: Febrero 28, 2019; disponible en línea: Septiembre 30, 2019. 